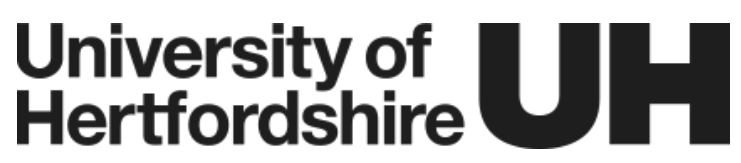

\section{Citation for the published version:}

Mansouri, M., Hassaine, S., Larbi, M., Bey, M., Allaoui, T., Denai, M., \& Moudjahed, M. (2019). New Hybrid Sensorless Speed of a Non-Salient Pole PMSG Coupled to Wind turbine Using a Modified Switching Algorithm. ISA Transactions.

DOI: 10.1016/j.isatra.2019.04.024

Document Version: Accepted Version

This manuscript is made available under the CC-BY-NC-ND license https://creativecommons.org/licenses/by-nc-nd/4.0/

\section{Link to the final published version available at the publisher:}

\section{https://doi.org/10.1016/j.isatra.2019.04.024}

\section{General rights}

Copyright $\odot$ and Moral Rights for the publications made accessible on this site are retained by the individual authors and/or other copyright owners.

Please check the manuscript for details of any other licences that may have been applied and it is a condition of accessing publications that users recognise and abide by the legal requirements associated with these rights. You may not engage in further distribution of the material for any profitmaking activities or any commercial gain. You may freely distribute both the url (http://uhra.herts.ac.uk/) and the content of this paper for research or private study, educational, or not-for-profit purposes without prior permission or charge.

\section{Take down policy}

If you believe that this document breaches copyright please contact us providing details, any such items will be temporarily removed from the repository pending investigation.

\section{Enquiries}

Please contact University of Hertfordshire Research \& Scholarly Communications for any enquiries at rsc@herts.ac.uk 


\section{Accepted Manuscript}

New hybrid sensorless speed of a non-salient pole PMSG coupled to wind turbine using a modified switching algorithm

Mansouri Mohamed, Hassaine Said, Larbi Mhamed, Bey Mohamed, Allaoui Tayeb, Denai Mouloud, Moudjahed Mohamed

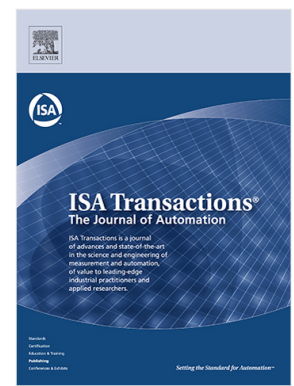

PII: $\quad$ S0019-0578(19)30198-3

DOI: $\quad$ https://doi.org/10.1016/j.isatra.2019.04.024

Reference: ISATRA 3184

To appear in: ISA Transactions

Received date: 23 July 2018

Revised date: 17 April 2019

Accepted date : 24 April 2019

Please cite this article as: M. Mohamed, H. Said, L. Mhamed et al., New hybrid sensorless speed of a non-salient pole PMSG coupled to wind turbine using a modified switching algorithm. ISA

Transactions (2019), https://doi.org/10.1016/j.isatra.2019.04.024

This is a PDF file of an unedited manuscript that has been accepted for publication. As a service to our customers we are providing this early version of the manuscript. The manuscript will undergo copyediting, typesetting, and review of the resulting proof before it is published in its final form. Please note that during the production process errors may be discovered which could affect the content, and all legal disclaimers that apply to the journal pertain. 
New Hybrid Sensorless Speed of a Non-Salient Pole PMSG Coupled to Wind Turbine Using a Modified Switching Algorithm.

\section{Author names:}

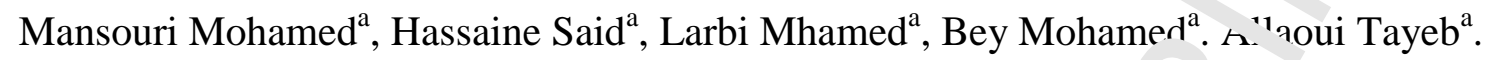

Denai Mouloud ${ }^{\mathrm{b}}$, Moudjahed Mohamed ${ }^{\mathrm{a}}$

\section{Author Emails:}

Mohamed.mansouri@univ-tiaret.dz, Mohamed.bey@univ-tiaret.dz allaoui_tb@yahoo.fr s_hassaine@y thor... larbi_mh@yahoo.fr, m.denai@herts.ac.uk

moudjahedm@yahoo.fr

\section{$\underline{\text { Author Affiliations: }}$}

${ }^{\text {a }}$ Laboratory of Energy Engineering and Computer _ Ingineering, IBN Khaldoun University, Tiaret, Algeria.

${ }^{\mathrm{b}}$ School of Engineering and Technology Inive sity of Hertfordshire, Hatfield, UK 


\section{Highlights}

- A new hybrid observer is proposed to estimate the position and speed of a non-salient pole PMSG driven by a wind turbine.

- The hybrid structure combines two observers and allow the ss ter to operate safely over a wide speed range without losing control.

- The proposed switching algorithm between the two ou rvers is based on the weighting coefficients method, which is characteri ed by simplicity and a low computation time.

- Speed control is based on fuzzy logic to provide ru'ustness and performance at low speeds.

- The proposed hybrid observer and cuntrol scheme are implemented in Matlab/Simulink and several simulation cenarios are presented to demonstrate the performance of the wind power sretem. 


\title{
New Hybrid Sensorless Speed of a Non-Salient Pole PMSG Coupled to Wind Turbine
}

\section{Using a Modified Switching Algorithm}

\begin{abstract}
The paper focuses on the design of position and speed observers for the ${ }^{-t}$ or of a non-salient pole permanent magnet synchronous generator (NSPPMSG) coup- $r$ to a wind turbine. With the random nature of wind speed this observer is required $t$, prov1 le a position and speed estimates over a wide speed range. The proposed hybric str _ $\_$re combines two observers and a switching algorithm to select the appropriate obser,$r^{r}$ based on a modified weighting coefficients method. The first observer is a higheı rde $_{\text {s }}$ iding mode observer (HOSMO) based on modified super twisting algorithm (STA, with correction term and operates in the medium and nominal wind speed ranges. The st $r$ nd observer is used in the low speed range and is based on the rotor flux estimation and $t^{2}$ cuntrol by injecting a direct reference current different to zero. The stability of each obse ver has been successfully assessed using an appropriate Lapunov function. Th simul tion results obtained show the effectiveness and performance of the proposed ob erv $\mathrm{r}$ ard control scheme.
\end{abstract}

\section{Keywords:}

Non-salient PMSG, st nd s one wind turbine, high-order sliding mode, super-twisting algorithm, fuzzy logi - co itro'

\section{Introduction}

Currently, wir ${ }^{-1}$ enc. $y$ is among the fastest growing renewable energy sources worldwide and wind pow ${ }^{2}$ in instry has experienced a rapid development over the last decade. Wind energy con $`$ sions systems (WECS) with various wind turbine configurations and generator types have been extensively studied and the doubly-fed induction generator (DFIG) and squirrel-cage induction generators (SCIG) have been the most commonly used generator 
technologies. In recent years, variable-speed WECS based on permanent magnet synchronous generators (PMSG) are becoming increasingly popular because of their advantages such as self-excitation, higher efficiency and improved reliability [1].

The field-oriented control (FOC) concept is often used to produce a df cou' led control of the torque and flux in the machine. To ensure a good operation at varjable . need, FOC requires continuous and accurate information of the rotor position and ${ }_{1}^{\circ} \dot{d}$. Therefore, either an encoder placed on the machine shaft or a programmed obsel rer is 1 ecessary to acquire this information. In general, sensor-based encoders for positio $/$ an $^{r^{\prime}} \mathrm{s}_{\mathbf{1}}$ 'eed have several drawbacks including hardware complexity, cost, sensitivity to exte. -al tactors such as vibration and temperature [2, 3]. Hence, extensive research th lu wsed on the design of software observers. These observers can be broadly classin $-d$ into three categories according to the operating regime of the machine. The observ ${ }^{*}$ of the first family are operated only at nominal and medium speed, they are based on he nachine standard model. The authors in [4] used the electromotive forces (EMF) to esthuate the rotor position. In case of noise, an extended Kalman filter (EKF) inter 'enes [^], which increases the calculation time. The model reference adaptive system $\left(\mathrm{M}^{\prime}<\mathrm{A}^{r}\right)$ ir another popular observer based on the machine parameters $[6,7]$. Robust $\mathbf{n},-$ inear observers based on sliding-mode have been extensively used in recent years $[8$, his observer is simple and robust against perturbations and parametric variations Howev,r, this observer requires a low-pass filter, which introduces a delay and reduces the 'ase margin of the system. Moreover, it needs an additional compensation of he $\mathfrak{p}$ sition $[9,10]$. The presence of chattering phenomenon can also deteriorate $\mathrm{t}$ ie per ormance of the mechanical systems because of excessive energy consumpt $t_{1} n$ un can lead to instability of the system [11]. To reduce the chattering phenomenon, it is necessary to shift the problem of discontinuity due to the switching element of the law in sliding regime on higher-order sliding variable derivatives. In [12] and [13], a super-twisting sliding mode observer (STSMO) was introduced to reduce the chattering. In 
the presence of bounded perturbations, the super-twisting algorithm (STA) gives better results and faster convergence $[14,15]$. In [16], it was shown that the STA cannot handle linearly growing perturbations and loses convergence. Hence, a modified STA is $\mathrm{ro}_{r}$ 'sed in $[9,17]$ to compensate this disturbance type, however, the performance at low ,pee $\downarrow$ is not addressed in the paper.

The second category are the low-speed observers and most $\iota^{c}$ nese are based on the anisotropic effects. These observers can be classified accordiı $g$ to th type of injected signal: (i) High-frequency rotating signal injection which can $b$ cla sied into $\alpha-\beta$ frame rotating injection $[18,19,20]$ or $d-q$ frame rotating injection $[2,2<]$. It has been shown that the current injection requires the current regulators ba. dwa... to be greater than the injection frequency and the voltage signal injection was he most commonly used method. Pulsating high-frequency signal injection when an the same way, the injected signal is a voltage [23] or a current [24] except that it is . ligıled with the d-axis or the estimated q-axis. Recently, in [25] the authors propose a new ıigh-frequency (HF) pulsating signal injection into the stationary reference frame $\gamma-\beta)$. $\mathrm{n}[26]$, the injected current pulses generate a noise which can reduce the accuracy of $t^{\prime}$ 'e e timation, the authors in [27] proposed using the two previous types, however, a - independent of the machine parameters but produces more losses. In [28], it was sh Jw that the precision of the position estimation is not satisfactory enough when using th : indire : flux detection by On-line Reactance Measurement (INFORM) method. A square wave : ,nal injection [29], and arbitrary injection [30] are other types of injected carrier $s_{\llcorner}$-nal. njection methods add a current to the machine and therefore cause heating and $\mathrm{r}$ bratiol s during high-speed phases [31].

The obse er ve the last category are designed for wide speed range operation: Two approaches exist. The first consists of using two observers in a hybrid configuration with a transition algorithm. A hybrid structure, consisting of a flux observer with a signal-injection technique has been proposed in $[20,32]$. However, at very low speed the dynamic 
performance is not satisfactory and is not presented in sufficient details. In [21], the $d-q$ frame persistent HF rotating carrier injection algorithm and a sliding mode observer (SMO) are combined by a weighting function, however, the direct torque contr / ( ( $2 \mathrm{TC}$ ) produces high torque and flux ripples. In [31], the High frequency signal injectir n (J FSI) technique is associated with an adaptive SMO but this configuration requires a hana- ${ }_{\mathbf{1}}$ ass (BPF) and lowpass filter (LPF). A combination of a HF injection technique and a $n$ del-based technique are presented by [33] using a weighted average method. The au hors in [22] proposed a hybrid position estimation combining the HFSI method with a las ${ }^{*} \sim$ SMO, however, the major drawback is the presence of LPF. In [3, 22, 23], a weight $\mu_{-}-$coefficients switching method is used. It is worth noting that all these observer types : ave $\_\sim \sim n$ proposed only for synchronous machines with non-uniform air gap. The observer, $n$ the other hand, operates directly over a wide speed range and does not need a transition . Is, orithm [34, 41].

In this paper, a new hybrid observer is propo ${ }^{-d}$ ior the estimation of the rotor position and speed of a non-salient pole PMSG driven by a wind turbine and operating over a wide speed range. The HOSM observer is bas $\cdot d$ on $c$ modified super-twisting algorithm. The observer gains are designed to compen ate ine arly growing perturbations. This proposed observer structure is able to extract $\ldots$ mechanical effect (i.e. position and speed) at nominal and medium wind speed. Ho / ev r, these observers are mainly dependent on the amplitude of the EMF, which explains ihe dey.adation of the estimation performance at low speeds. The best known estimation mothu'c at low speed among the existing ones are only suitable for salient pole machines. Fc this eason, another type of observer is proposed in [34] and adapted with the non-salie t pole 'MSG. The speed was determined from the quadrature flux and regulated

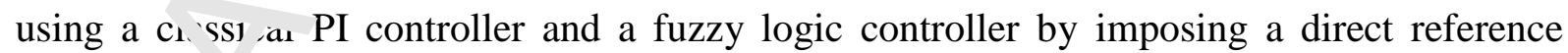
current different from zero. The advantage of this observer structure is that it does not degrade the performance of the generator at high speeds. The introduction of weighting factors is necessary to select and combine these two observers in order to improve the signal of the 
transition region according to the operating speed of the generator. The stability of each observer is established by Lyapunov method with an appropriate Lyapunov function. The performance of the proposed hybrid observer is evaluated via simulations Wn' PI and fuzzy logic controllers at low speed and a comparison between the class ${ }^{*}$ al ind the proposed observers is presented to demonstrate the effectiveness of the hybrid stı - ture and switching strategy. This paper is organized in six sections. Section 2 descric ${ }^{\text {ar }}$ the models of the wind turbine and PMSG. Section 3 presents the stator-side contı $)$ sche ne implemented in the model. The detailed derivation of the hybrid observer an $\mathrm{sw}$ : . $\therefore$ ing algorithm are presented in Section 4 and 5 respectively. Section 6 presents th simulation results. Finally, the conclusions on this paper are summarized in Section ${ }^{?}$ ?

\section{Modeling of the Wind Power Generation Sysu n}

The wind power system considered in this st $\mathrm{f}$ consists of a wind turbine, a PMSG, a converter composed of a rectifier and an inv r rte separated by a dc bus and a three phase current filter connected to grid as depicted in rigure 1 . The wind turbine drives the PMSG at a variable-speed through a gearbox [ 5]. Tht focus of this study is on the selected part (dottedline) of the Figure 1 which repr $\operatorname{sen}^{\prime} s$ th $^{\wedge}$ wind conversion system.

\subsection{Turbine Model}

The mechanical power ar pea ing at the rotor of the wind turbine driving a generator through a gearbox can be writte as folı ws:

$P_{m}=\frac{1}{2} C_{p}(\lambda) \rho \pi^{r} \cdot-V_{w}^{3}$

Where $\rho$ is th $\_$air density $\left(\mathrm{kg} / \mathrm{m}^{3}\right), R$ is the blade radius $(\mathrm{m}), V_{w}$ is the wind speed $(\mathrm{m} / \mathrm{s})$ and $C_{p}$ is the power uurficient. The value of $C_{p}$ depends on the tip speed ratio $(\lambda)$, the pitch angle $(\beta)$ and the 1 sitive constants $c_{1}$ to $c_{6}$ [36].

$C_{p}=c_{1}\left(\frac{c_{2}}{\lambda_{i}}-c_{3} \beta-c_{4}\right) e^{\frac{-c_{5}}{\lambda_{i}}}+c_{6} \lambda$ 
With

$\frac{1}{\lambda_{i}}=\frac{1}{\lambda+0.08 \beta}-\frac{0.035}{\beta^{3}+1}$

And $C_{p}=0.38, \beta=0, \lambda_{\text {opt }}=6.1, c_{1}=0.22, c_{2}=116, c_{3}=0.4, c_{4}=5, c_{\llcorner}=12.5, c_{6}=0$

The role of the gearbox coupling the wind turbine to the generatos ${ }^{\circ}$ to dapt the turbine speed $\omega_{t}$ with the generator speed $\omega_{g}$. It is modeled as follows [4 $\left[4^{-}\right.$:

$\omega_{t}=\frac{1}{G} \omega_{g}$

$\mathcal{T}_{g}=\frac{1}{G} \mathcal{T}_{t}$

Where $\mathcal{T}_{t}$ is the turbine torque, $\mathcal{T}_{g}$ is the generator torqu and $G$ is the gearing ratio.

\subsection{The model of PMSG}

The PMSG model in the stationary reference fra ne $(u, \beta)$ attached to the stator, is written as:

$\left\{\begin{array}{l}v_{\alpha}=-R_{s} i_{\alpha}-L_{s} \frac{d i_{\alpha}}{d t}+e_{\alpha} \\ v_{\beta}=-R_{s} i_{\beta}-L_{s} \frac{d i_{\beta}}{d t}+e_{\beta}\end{array}\right.$

Where $v_{\alpha \beta}$ are stator terminal vo'tagt,$R$, and $L_{s}$ denote the stator resistance and inductance respectively, $i_{\alpha \beta}$ represent the staı " $\mathrm{cr}$ irents and $e_{\alpha, \beta}$ are the back EMFs which are given by:

$\left\{\begin{array}{l}e_{\alpha}=-\omega_{r} \varphi_{p m} \sin \theta \\ e_{\beta}=\omega_{r} \varphi_{p m} \cos \theta\end{array}\right.$

Where $\omega_{r}, \theta$ and,$_{p r}$ are the rotor speed, rotor position and magnetic flux linkage respectively. The 1 ectromagnetic torque and the mechanical equation can be written as:

$\mathcal{T}_{\text {em }}=n_{p} K_{e}\left(i, \cos 乞 \quad i_{\alpha} \sin \theta\right)$

$\frac{d \omega_{r}}{d t}=n_{i} K\left(i_{R} \cos \theta-i_{\alpha} \sin \theta\right)-\frac{f_{v}}{\mathcal{J}} \omega_{r}-\frac{\mathcal{T}_{t}}{\mathcal{J}}$

Where $\mathcal{J}$ is the inertia, $f_{v}$ is the viscous friction and $\mathcal{T}_{t}$ is the mechanical torque.

$K_{e}=\sqrt{\frac{3}{2}} \varphi_{p m}$ is the EMF constant and $n_{p}$ is the pole pair number [37]. 


\section{Stator-Side Converter Control}

The basic structure of the control scheme is to decompose the stator current into two parts, one component controls the flux and the other component controls the $\mathrm{irl}_{4}$ 'e. The speed regulation is used to control the torque at the same time to extract the nax mum power from the turbine using a maximum power point tracking (MPPT) strateov. The speed reference depends on the wind speed and the optimal tip speed ratio is given :

$$
\omega^{*}=\frac{\lambda_{o p t} \times V_{w}}{R}
$$

Were $\lambda_{\text {opt }}$ is the optimal tip speed ratio and $V_{w}$ is the wind speed $\mathrm{m} / \mathrm{s}$ ).

Figure 2 shows the various control blocks on the gє. rator ide [7]. The estimated speed is regulated through the external loop while the internal 1 ${ }_{\text {r }}$ controls the $I_{q}$ current component.

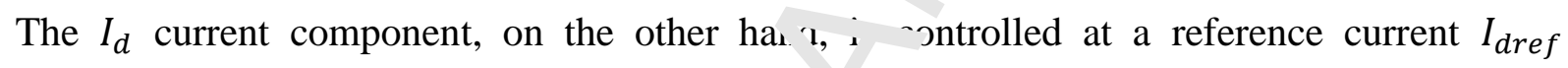
$\left(I_{\text {dref }} \neq 0\right.$ at low speed and $I_{\text {dref }}=0$ othe $w_{\text {: }}$ ). In contrast, the current $I_{\text {qref }}$ along the quadrature axis $q$ is extracted from the spe ${ }^{\top}$ regulator output, which is proportional to the electromagnetic torque. The output, of cu rent regulators are combined with the decoupling block to generate the reference v slta ,es $\left(v_{\alpha r e f}, v_{\beta r e f}\right)$. The space vector modulation (SVM) is applied to transform these re erences voltages into pulse width modulation (PWM) signals to control the inverter swit $1 \mathrm{tc}$. The first observer (HOSM) estimates the rotor position and velocity from voltages and cu rents measurements in a fixed reference frame $(\alpha, \beta)$, while the second observer estima ${ }^{+}$le mechanical variables from voltages and currents in the rotating frame $(d, q)$. The witch ng between the two observers is achieved by a transition bridge.

\section{Design o the $O$ iservers}

\subsection{Supe - I v : ':ng Sliding Mode Observer}

This observer is based on the classical variable structure theory. The aim is to generate a second order sliding regime on a correctly chosen surface to constrain the system trajectories to be evaluated after a finite time on the set $\{S=\dot{S}=0\}$. It is characterized by the rotation of 
the trajectories around the origin of the phase diagram [38, 39].

The standard model of STA can be represented by:

$\dot{x}_{1}=-k_{1}\left|\bar{x}_{1}\right|^{1 / 2} \operatorname{sign}\left(\bar{x}_{1}\right)+x_{2}+\delta_{1}\left(x_{1}, t\right)$

$\dot{x}_{2}=-k_{2} \operatorname{sign}\left(\bar{x}_{1}\right)+\delta_{2}\left(x_{2}, t\right)$

Where $x_{i}$ are the state variables, $\bar{x}_{i}$ are the errors between state variabı $k_{i}$ represent the sliding coefficients, $\delta_{i}$ are perturbation terms and $\operatorname{sign}(\cdot)$ is the sig. unction.

If the following conditions are satisfied, then STA-SMO conve rges in a finite time:

$$
\begin{aligned}
& k_{1}>2 \mathfrak{h}_{1}, \quad k_{2}>k_{1} \frac{5 \mathfrak{h}_{1} k_{1}+4 \mathfrak{h}_{1}^{2}}{2\left(k_{1}-2 \mathfrak{h}_{1}\right)} \\
& \left|\delta_{1}\right| \leq \mathfrak{h}_{1} \sqrt{\left|x_{1}\right|}, \quad \delta_{2}=0
\end{aligned}
$$

Where $\mathfrak{h}_{1}$ is a positive constant $[16,41]$.

\subsection{Higher-Order Sliding Mode Observer for P'ASG}

This observer estimates the mechanical quantı: es 'speed, position) from the EMFs estimation. The only inputs data provided to this ou erver are the stator currents and voltages measurements. To apply the STSN algoril im to the PMSG, the model of the machine given in (5) should be modified as foll $\mathrm{Jws}$

$$
\begin{aligned}
& \frac{d \hat{\imath}_{\alpha}}{d t}=\frac{-R_{s}}{L} \hat{\imath}_{\alpha}-\frac{1}{L} v_{\alpha}+\frac{1}{L} 1_{\alpha}(L) \\
& \frac{d \hat{\imath}_{\beta}}{d t}=\frac{-R_{s}}{L} \hat{\imath}_{\beta}-\frac{1}{L} v_{\beta}+\frac{-}{L} u_{\beta}(t)
\end{aligned}
$$

Where ^ denotes esti. ^at d $\mathrm{v}$.lue, and $L=L_{d}=L_{q}$.

Comparing with (5) an (6), it can be noticed that $\frac{-R_{S}}{L} \hat{\imath}_{\alpha}-\frac{1}{L} v_{\alpha}$ and $\frac{-R_{S}}{L} \hat{l}_{\beta}-\frac{1}{L} v_{\beta}$ in the PMSG volta e equi ions are considered as perturbation terms. $u_{\alpha, \beta}(t)$ are the robust terms based on . ve ¿ - ical STA [41] defined as :

$$
u_{\alpha}(t)=-\mathcal{K}_{1}\left|\mathcal{S}_{\alpha}(t)\right|^{\frac{1}{2}} \operatorname{sign}\left(\mathcal{S}_{\alpha}(t)\right)-\mathcal{K}_{2} \int \operatorname{sign}\left(\mathcal{S}_{\alpha}(t)\right) d t
$$

Similarly, $u_{\beta}(t)$ can be obtained by replacing $\alpha$ with $\beta$. 
The sign function limit can slow down the trajectories of the system when they tend to move away from the origin. Therefore, some linear terms are introduced to improve the convergence rate and make the sliding surface insensitive to the increasing pe rrbations. The following new correction terms are proposed [17,9]:

$u_{\alpha}(t)=-\mathcal{K}_{1} \psi_{1}\left(\mathcal{S}_{\alpha}(t)\right)-\mathcal{K}_{2} \int \psi_{2}\left(\mathcal{S}_{\alpha}(t)\right) d t$

In the same way, $u_{\beta}(t)$ is obtained by replacing $\alpha$ with $\beta$.

The sliding surfaces are chosen as:

$\mathcal{S}_{\alpha}(t)=\hat{\imath}_{\alpha}-i_{\alpha}$

$\mathcal{S}_{\beta}(t)=\hat{\imath}_{\beta}-i_{\beta}$

Where $\hat{\imath}_{\alpha}$ and $\hat{l}_{\beta}$ are the estimated currents, $i_{\alpha}$ ar. ${ }^{\prime} i_{\beta}$ un the actual currents.

To compensate for linearly increasing pe urr aiuns, the nonlinear stabilizing terms $\psi_{1}\left(\delta_{\alpha \beta}(t)\right), \psi_{2}\left(\delta_{\alpha \beta}(t)\right)$ are introduced anc aı defined by:

$\psi_{1}\left(\mathcal{S}_{\alpha}(t)\right)=\mathcal{S}_{\alpha}(t)+K_{3} \sqrt{\left|\mathcal{S}_{\alpha}(t)\right|} \tanh \left(\mathcal{S}_{\alpha}(t)\right)$

$\psi_{2}\left(\mathcal{S}_{\alpha}(t)\right)=\mathcal{S}_{\alpha}(t)+\frac{1}{2} \mathcal{K}_{4}^{2} \tanh \left(\mathcal{S}_{\alpha}(\imath)+\frac{3}{2} \mathcal{K}_{4} \sqrt{\left|\mathcal{S}_{\alpha}(t)\right|} \tanh \left(\mathcal{S}_{\alpha}(t)\right)\right.$

Similarly, the functions $\psi_{1}\left(r_{\beta}(t) ;{ }^{a} \mathrm{~d} \psi_{2}\left(\mathcal{S}_{\beta}(t)\right)\right.$ can be obtained by replacing $\mathcal{S}_{\alpha}(t)$ with $\mathcal{S}_{\beta}(t)$ in $(17 \mathrm{a}, 17 \mathrm{~b})[17]$.

Where $\mathcal{K}_{1}, \mathcal{K}_{2}, \mathcal{K}_{3}$ and $\mathcal{K}_{4} \omega^{\bullet}$ design positive constants. The gain $\mathcal{K}_{1}$ can cause saturation when it is high. $\mathcal{K}_{3}$ anı $\mathcal{K}_{2}$ are chosen to improve the stability in finite time and to reject the uncertainties effe ${ }^{\top}$. Th $\mathrm{T}$ gain $\mathcal{K}_{4}$ should be adjusted achieve a balance between robustness and chatterir, $\mathcal{K}_{1}{ }^{\prime}{ }_{1}\left(\mathcal{S}_{\alpha}(t)\right)$ is utilized to improve convergence time when trajectories are far from th' $\mathrm{O}^{\prime}$, gitl, and $\mathcal{K}_{2} \psi_{2}\left(\mathcal{S}_{\alpha}(t)\right)$ will dominate the perturbation [9].

\subsubsection{Sliding Mode Stability}

To analyze the stability, the time derivatives of the sliding surface are determined from (5) and (13) as follow [40]: 


$$
\begin{aligned}
& \dot{\mathcal{S}}_{\alpha}=\frac{-R_{s}}{L} \hat{\mathcal{S}}_{\alpha}-\frac{1}{L} e_{\alpha}+\frac{1}{L} u_{\alpha}(t) \\
& \dot{\mathcal{S}}_{\beta}=\frac{-R_{s}}{L} \hat{\delta}_{\beta}-\frac{1}{L} e_{\beta}+\frac{1}{L} u_{\beta}(t)
\end{aligned}
$$

Assumption 1: There exist two constants $\varrho_{\alpha, \beta}$ such that the disturbances ' $\sim$ ' 1 s are bounded as follows:

$$
\begin{aligned}
& \left|\dot{e}_{\alpha}\right| \leq \varrho_{\alpha} \\
& \left|\dot{e}_{\beta}\right| \leq \varrho_{\beta}
\end{aligned}
$$

Since $\omega_{s}, \mathcal{T}_{l}, e_{\alpha, \beta}$ and $i_{\alpha, \beta}$ are continuous on a comr $\mu$ set, the condition (14) is not restrictive.

Theorem 1: According to Assumption 1, the origin $v_{i} \mathrm{cv}^{\mathrm{s}} . \mathrm{em}$ in $(18 \mathrm{a}, 18 \mathrm{~b})$ is robust and globally stable equilibrium point in a finite tin. Further, the estimation of the unknown input/perturbation e $(\mathcal{S}, \mathrm{t})$ is given by $\mathcal{K}_{2} \int_{0}^{\mathrm{t}} \psi_{2}\left(\mathrm{c}^{\cdot} \boldsymbol{t}^{\dagger}\right) \mathrm{dt}$ after a finite-time.

Proposition 1: Consider the matrix $\mathcal{A}_{0}$ :

$\mathcal{A}_{0}=\frac{1}{L}\left[\begin{array}{cc}-\left(\mathcal{K}_{1}+R_{S}\right) & L \\ -\mathcal{K}_{2} & 0\end{array}\right]$

Where $\mathcal{K}_{1}>0$ and $\mathcal{K}_{2}>0$, so th at $, \mathcal{A}_{0}:$ Hurwitz matrix.

From $(17 \mathrm{a}, 17 \mathrm{~b})$ and $\left(18 \mathrm{a}, 1^{\mathrm{r}}\right)$, the $-\mathbf{v}$, tem can be represented as [9]:

$$
\begin{aligned}
& \dot{\mathcal{S}}_{1}=\mathcal{S}_{2}-\left(\frac{\mathcal{K}_{1}+R_{s}}{L}\right)\left(\mathcal{S}+\mathcal{Y}_{4}\left[\mathcal{S}_{1}\right]^{1 / 2} \operatorname{sign}\left(\mathcal{S}_{1}\right)\right) \\
& \dot{\mathcal{S}}_{2}=-\frac{\mathcal{K}_{2}}{L}\left(\mathcal{S}_{1}+\frac{\mathcal{K}^{2}}{2} \mathrm{si}, \mathrm{n}\left(\kappa_{1}\right)+3 \frac{\mathcal{K}_{4}}{2}\left\lceil\mathcal{S}_{1}\right\rceil^{1 / 2} \operatorname{sign}\left(\mathcal{S}_{1}\right)\right)+\frac{\dot{e}_{\alpha, \beta}}{L}
\end{aligned}
$$

With

$$
\mathcal{S}_{1}=\mathcal{S}_{\alpha, \beta}, \delta_{\dot{L}}=\left(\begin{array}{c}
1 \\
\frac{1}{L}
\end{array}\right)\left[e_{\alpha, \beta}-\mathcal{K}_{2} \int \psi_{2}\left(\mathcal{S}_{1}(t)\right) d t\right]
$$

From (13a) a 'd (21a):

$$
\mathcal{K}_{4}=\mathcal{K}_{1} \mathcal{K}_{3} /\left(\mathcal{K}_{1}+R_{S}\right)
$$


Proof 1 [16]: based on (17b), if $\mathcal{S}_{\alpha, \beta}=\dot{\mathcal{S}}_{\alpha, \beta}=0$, then $\left|\phi_{2}(s)\right| \geq \mathcal{K}_{4}^{2} / 2$, and one gets:

$|\dot{e}(\mathcal{S}, t)| \leq\left|\psi_{2}(\mathcal{S})\right|$, so $\mathcal{K}_{4} \geq \sqrt{2 \varrho_{\alpha, \beta}}$

and

$\mathcal{K}_{3} \geq \sqrt{2 \varrho_{1,2}}\left(\mathcal{K}_{1}+R_{s}\right) / \mathcal{K}_{1}$

To prove the stability and the convergence in finite time, the foll ' $\mathrm{g}$ Lyapunov function in quadratic form is used [14]:

$V(\xi)=\xi^{T} \mathcal{F} \xi$

Where $\mathcal{F}$ is a constant, symmetric and positive definite sc 'יition of $V$ Such that $\mathcal{F}=\mathcal{F}^{T}=$ $\left[\begin{array}{cc}\Gamma+4 \varepsilon^{2} & -2 \varepsilon \\ -2 \varepsilon & 1\end{array}\right], \Gamma>0$ and $\varepsilon>0$ and with a new staı vector:

$\xi=\left[\begin{array}{c}\mathcal{S}_{1}+\mathcal{K}_{4}\left[\mathcal{S}_{1}\right]^{1 / 2} \operatorname{sign}\left(\mathcal{S}_{1}\right) \\ \mathcal{S}_{2}\end{array}\right]=\left[\begin{array}{l}\xi_{1} \\ \xi_{2}\end{array}\right]$

The derivative of the Lyapunov function is:

$\dot{V}(s)=\dot{\xi}^{T} \mathcal{F} \xi+\xi^{T} \mathcal{F} \dot{\xi}$

And the standard inequality for qua ${ }^{\top}$ ratic $f$,rms is given by:

$\Gamma_{\min }\{\mathcal{F}\}\|\xi\|^{2} \leq \xi^{T} \mathcal{F} \xi \leq \Gamma_{\max }\{-\} \mid\left\|^{1},\right\|^{2}$

From (26), the Euclidean nc $\mathrm{n}, \mathrm{f} \xi$ is:

$\|\xi\|^{2}=\mathcal{S}_{1}^{2}+2 \mathcal{K}_{4}\left|\mathcal{S}_{1}\right|^{3 / \cdot}+\mathcal{c}_{4}^{2}\left|\mathcal{S}_{1}\right|+\xi_{2}^{2}$

From (28), note that + e i equality :

$\|\xi\| \leq \frac{V^{1 / 2}(\mathcal{S})}{\Gamma_{\min }^{1 / 2}\{\mathcal{F}\}}$

Using (29), $\left.\right|_{1} ^{\succ} \|$ can se written as:

$\|\xi\|^{2} \geq \mathcal{K}_{4}^{?}\left|\mathcal{S}_{2}\right|$

While $\mathcal{K}_{4}>0$, then:

$-\frac{\mathcal{K}_{4}}{\|\xi\|} \geq-\left|\mathcal{S}_{1}\right|^{-1 / 2}$ 
When expressed along the trajectories of the system, the derivative of the Lyapunov function becomes:

$\dot{V}=\left(1+\frac{\mathcal{K}_{4}}{2}\left|\mathcal{S}_{1}\right|^{-1 / 2}\right) \xi^{T}\left(\mathcal{A}_{0}^{T} \mathcal{F}+\mathcal{F} \mathcal{A}_{0}\right) \xi+2 \xi^{T} \mathcal{F}\left[\begin{array}{c}0 \\ \dot{e}\end{array}\right]$

Where $\mathcal{F}$ and $\mathcal{H}$ are related by the algebraic Lyapunov equation:

$-\mathcal{H}=\mathcal{A}_{0}^{T} \mathcal{F}+\mathcal{F}_{\mathcal{A}_{0}}=-\left[\begin{array}{ll}\mathcal{H}_{1} & \mathcal{H}_{2} \\ \mathcal{H}_{2} & \mathcal{H}_{3}\end{array}\right]$

$\mathcal{H}=\mathcal{H}^{T}>0$ is arbitrary, symmetric and positive definite $\mathrm{m}$. trix an ? represent the feedback gain matrix. From proof 1, one can note that:

$$
\begin{aligned}
\dot{V} & \leq\left(1+\frac{\mathcal{K}_{4}}{2}\left|\mathcal{S}_{1}\right|^{-1 / 2}\right)\left(\xi^{T}\left(\mathcal{A}_{0}^{T} \mathcal{F}+\mathcal{F} \mathcal{A}_{0}\right) \xi+2 \xi^{T} \mathcal{F}\left[\begin{array}{l}
0 \\
\xi_{1}
\end{array}\right]\right) \\
& \leq-\left(1+\frac{\mathcal{K}_{4}}{2}\left|\mathcal{S}_{1}\right|^{-1 / 2}\right) \xi^{T} \mathcal{H} \xi
\end{aligned}
$$

From (28), it implies that:

$\dot{V} \leq-\left(1+\frac{\mathcal{K}_{4}}{2}\left|\mathcal{S}_{1}\right|^{-1 / 2}\right) \Gamma_{\min }(\mathcal{H})\|\xi\|^{2}$

With

$$
\left\{\begin{array}{l}
\mathcal{H}_{1}=2\left(\frac{\mathcal{K}_{1}+R_{S}}{L}\right)\left(\Gamma+4 \varepsilon^{2}\right)-4\left(\frac{\ddots}{\mathcal{C}}-1\right) \\
\mathcal{H}_{2}=-2 \varepsilon\left(\frac{\mathcal{K}_{1}}{L}+\frac{R_{S}}{L}\right)+\left(\frac{\mathcal{P}}{L}+1\right)-\left(\Gamma+4 \varepsilon^{2}\right) \\
\mathcal{H}_{3}=4 \varepsilon
\end{array}\right.
$$

For $\mathcal{H}$ to be positive, $\mathcal{K}_{2}$ a. $1 \mathcal{K}_{1}$ should be such that:

$$
\begin{aligned}
& \mathcal{K}_{2}=L\left(\Gamma+4 \varepsilon^{2}+\frac{\omega}{L}\left({ }^{\prime}{ }_{1}\left\ulcorner R_{S}\right)\right)\right. \\
& \mathcal{K}_{1}>-R_{S}+\frac{1}{\Gamma}\left(2 \varepsilon L_{1}-2 \varepsilon L+8 L \varepsilon^{3}\right)
\end{aligned}
$$

Hence,

$\dot{V} \leq-\mu_{1} V^{1,}(\mathcal{S})-\mu_{2} V(\mathcal{S})$

With $\mu_{1}=\frac{\Gamma_{\min }(\mathcal{H})}{\Gamma_{\max }^{1 / 2}(\mathcal{F})} \frac{\mathcal{K}_{4}^{2}}{2}$ and $\mu_{2}=\frac{\Gamma_{\min }(\mathcal{H})}{\Gamma_{\max }(\mathcal{F})}$ 
Where $\Gamma_{\max }$ and $\Gamma_{\min }$ represents respectively the maximum and minimum singular values of matrix $\mathcal{F}[14,17,42]$.

\subsubsection{Convergence Time}

The solution of the differential equation (40) is given by :

$V(t)= \begin{cases}\left(V^{1 / 2}\left(\mathcal{S}_{\alpha, \beta}\left(t_{0}\right)\right)-\frac{\mu_{1}}{2} t\right)^{2} & \text { if } \mu_{1}=1, \quad,=\imath \\ e^{-\mu_{2} t}\left(V^{1 / 2}\left(\mathcal{S}_{\alpha, \beta}\left(t_{0}\right)\right)+\frac{\mu_{1}}{\mu_{2}}\left(1-e^{\frac{\mu_{2}}{2} t}\right)\right)^{2} & \text { if } \mu_{1} \geq 0, \cdot>0\end{cases}$

At time $t=0$ the system trajectory $(21 \mathrm{a}, 21 \mathrm{~b})$ starting at $\left.\mathcal{S}_{\alpha, \beta}{ }^{\prime} t_{0}\right)$ w' 1 converge to the origin $\left(\mathcal{S}_{\alpha, \beta}=0\right)$ in a finite time. When $\mu_{1}=1$ all trajectories $\mathrm{c} \mathrm{n}^{\mathrm{v}} \mathrm{vg} \mathrm{g}$ to the origin in finite time, and when $\mu_{1}=0$ the convergence is exponential. From (4) the convergence time can be written as [14. 16] :

$$
\begin{aligned}
& \text { T } \\
& = \begin{cases}\frac{2 V^{1 / 2}\left(\mathcal{S}_{\alpha, \beta}\left(t_{0}\right)\right)}{\mu_{1}} & \text { if } \mu_{2}=0 \\
\frac{2}{\mu_{2}} \ln \left(\frac{\mu_{2}}{\mu_{1}} V^{1 / 2}\left(\mathcal{S}_{\alpha, \beta}\left(t_{0}\right)\right)+1\right) & \text { if } \mu_{2}>0\end{cases}
\end{aligned}
$$

\subsubsection{Speed and Rotor Positior Es. '}

When STA-SMO reaches the en libr um point, this means that $\mathcal{S}_{\alpha, \beta}=\dot{\mathcal{S}}_{\alpha, \beta}=0$ in finite time, equation $(18 \mathrm{a}, 18 \mathrm{~b}) \mathrm{becom}$

$$
\begin{aligned}
& 0=-\mathcal{K}_{2} \int_{0}^{t} \psi_{2}\left(\mathcal{S}_{\alpha}(t)\right) d t \boldsymbol{\tau} \hat{o}_{\gamma} \\
& 0=-\mathcal{K}_{2} \int_{0}^{t} \psi_{2}\left(\mathcal{S}_{\beta}(t), d t+\hat{e}_{\beta}\right.
\end{aligned}
$$

Then, the est mated rack-EMF can be obtained as follows:

$$
\begin{aligned}
& \hat{e}_{\alpha}=K_{2} \int_{0}^{+} v_{-}\left(\partial_{\alpha}(t)\right) d t \\
& \hat{e}_{\beta}=K_{2} \int_{0}^{t} \psi_{2}\left(\delta_{\beta}(t)\right) d t
\end{aligned}
$$


Equation (6) shows that there is a relation between the EMF and the rotor position, so it is expressed by:

$\hat{\theta}_{1}=-\tan ^{-1}\left(\frac{\hat{e}_{\alpha}}{\hat{e}_{\beta}}\right)$

The expression of the estimated speed is deduced from the EMFs as fu'nws ? 7$]$ :

$\widehat{\omega}_{1}=\frac{1}{k_{e}} \sqrt{\hat{e}_{\alpha}^{2}+\hat{e}_{\beta}^{2}}$

\subsection{Design of the Second Observer}

The HOSMO described in the previous section is sui ${ }^{\prime}{ }^{\prime} \circ$ for medium and high speed sensorless operation, where the electromotive force (EMF) c $\eta$ be easily estimated. However, at low speed, this EMF is very small which leads to a noor estimation performance and the effects of inverter nonlinearity become severe $[16, \therefore 1]$. To alleviate this problem, another approach is proposed.

The approach adopted in this observer i zesen on the injection of a non-zero direct current. The principle is to force this current in the defective position, and this will force the position of the rotor to the incorrectly estim. ted position, and consequently the error between the actual and the estimated posit ${ }^{n}$ will be reduced [34]. The velocity is obtained from the imaginary part of the flux $\mathrm{IS}_{\mathrm{s}} \mathrm{\iota}_{\mathrm{c}}$ two control methods: a classical PI controller and a fuzzy logic controller as show in igure 3. The position is the integral of this velocity. The current and voltage are exprf sser with a complex space phasors as follow:

$$
\begin{aligned}
& i_{s}=\frac{2}{3}\left(i_{a}+a i_{b}+a^{2} i_{c}\right) \\
& v_{s}=\frac{2}{3}\left(v_{a}+x v_{b}+x^{2} v_{c}\right)
\end{aligned}
$$

$a$ and $a^{2}$ ar ne spatial operators, where $a=e^{j 2 \pi / 3}$. The flux equations are given by [43]:

$$
\left\{\begin{array}{l}
\dot{\varphi}_{s}=v_{s}-R_{s} i_{s} \\
\varphi_{s}=L i_{s}+\varphi_{r} \\
\varphi_{r}=\varphi_{m} e^{j \theta}
\end{array}\right.
$$


The voltage of the PMSG expressed in the coordinate related to the rotor is given by [34]:

$v_{s}^{(R)}=-R i_{s}^{(R)}-\left(S+j \omega_{2}\right) L i_{s}^{(R)}+j \omega \varphi_{m}$

Where $S$ is the time derivative operator. The voltage equation in the coordinal $y s t e m e^{j \widehat{\theta}_{2}}$ becomes:

$v_{s}^{(\widehat{R})}=-R_{s} i_{s}^{(\hat{R})}-\left(S+j \widehat{\omega}_{2}\right)\left(L i_{s}^{(\widehat{R})}-\varphi_{m} e^{j\left(\theta-\widehat{\theta}_{2}\right)}\right)$

Where $\widehat{\omega}_{2}=S \hat{\theta}$. Let $\tilde{\theta}=\widehat{\theta}_{2}-\theta$, thus:

$e^{j \widetilde{\theta}}=\cos \tilde{\theta}+j \sin \tilde{\theta}$

Substituting (52) into (51) gives after simplifications:

$\varphi_{m}-L i_{s}=\frac{1}{S+j \widehat{\omega}_{2}}\left(v_{s}+R_{s} i_{s}+\left(\omega \sin \tilde{\theta}+j\left(\omega \iota<\theta-\omega_{2}\right)\right) \varphi_{m}\right)$

The observer may take the following form:

$\hat{\varphi}_{r}-L i_{s}=\frac{1}{S+j \widehat{\omega}_{2}}\left(v_{s}+R_{S} i_{s}+\mathcal{Q}\right)$

By subtracting (53) from (54), the flux dynani - error is obtained as:

$\left.S\left(\hat{\varphi}_{r}-\varphi_{m}\right)=-j \widehat{\omega}_{2}\left(\hat{\varphi}_{r}-\varphi_{m}\right)+\right)+\left(\omega \sin \tilde{\theta}+j\left(\omega \cos \tilde{\theta}-\widehat{\omega}_{2}\right)\right) \varphi_{m}$

Where $\hat{\varphi}_{r}=\hat{\varphi}_{r d}+j \hat{\varphi}_{r q}$

Stabilizing with $\mathcal{Q}=-\mathcal{C}_{1}\left(\rho_{r_{u}}-\varphi_{m}\right)-\mathcal{C}_{2} \hat{\varphi}_{r q}$, equation (55) is decomposed into a real and an imaginary part:

$\left.S\left(\hat{\varphi}_{r d}-\varphi_{m}\right)=-\mathcal{C}_{1}, \hat{\varphi}_{r},-\varphi_{m}\right)+\widehat{\omega}_{2} \hat{\varphi}_{r q}+\omega \sin \tilde{\theta} \varphi_{m}$

$S \hat{\varphi}_{r q}=-\mathcal{C}_{2} \hat{\varphi}_{r q}-\omega_{2}\left(\hat{\jmath}_{r d}-\varphi_{m}\right)-\left(\widehat{\omega}_{2}-\omega \cos \tilde{\theta}\right) \varphi_{m}$

With $\hat{\varphi}_{r q}=-\psi_{m} \sin \sigma$, and $\hat{\varphi}_{r d}=\varphi_{m} \cos \tilde{\theta},(56,57)$ become :

$S\left(\hat{\varphi}_{r d}-\varphi_{m}\right)-{ }_{-1}\left(\hat{\varphi}_{r d}-\varphi_{m}\right)+\widehat{\omega}_{2} \hat{\varphi}_{r q}-\omega \hat{\varphi}_{r q}$

$S \hat{\varphi}_{r q}=-\mathcal{C}_{2} \hat{\imath}_{r q}-\widehat{\omega}_{2}\left(\hat{\varphi}_{r d}-\varphi_{m}\right)-\left(\widehat{\omega}_{2}-\omega\right) \varphi_{m}+\omega\left(\hat{\varphi}_{r d}-\varphi_{m}\right)$

Replacing $\widetilde{\omega}=\widehat{\omega}_{2}-\omega$ in $(58,59)$ gives:

$S\left(\hat{\varphi}_{r d}-\varphi_{m}\right)=-\mathcal{C}_{1}\left(\hat{\varphi}_{r d}-\varphi_{m}\right)+\widetilde{\omega} \hat{\varphi}_{r q}$ 
$S \hat{\varphi}_{r q}=-\mathcal{C}_{2} \hat{\varphi}_{r q}-\widetilde{\omega}\left(\hat{\varphi}_{r d}-\varphi_{m}\right)-\widetilde{\omega} \varphi_{m}$

\subsubsection{Stability Analysis}

The stability is assessed using the following Lyapunov function:

$\mathcal{P}=\frac{1}{2}\left(\left(\hat{\varphi}_{r d}-\varphi_{m}\right)^{2}+\left(\hat{\varphi}_{r q}\right)^{2}+\frac{1}{\sigma} \widetilde{\omega}^{2}\right)$

The time derivative of the Lyapunov function $\mathcal{P}$ is obtained wit', ar $\mu_{2}$ ement of equations $(60,61)$ and (62) as:

$\frac{d \mathcal{P}}{d t}=-\mathcal{C}_{1}\left(\hat{\varphi}_{r d}-\varphi_{m}\right)^{2}-\mathcal{C}_{2}\left(\hat{\varphi}_{r q}\right)^{2}+\widetilde{\omega}\left(-\varphi_{m} \hat{\varphi}_{r q}+\frac{1}{\sigma}-\frac{d \widetilde{\cdots}}{t}\right)$

If $\frac{d \widetilde{\omega}}{d t}=\sigma \varphi_{m} \hat{\varphi}_{r q}$, equation (63) becomes:

$\frac{d \mathcal{P}}{d t}=-\mathcal{C}_{1}\left(\hat{\varphi}_{r d}-\varphi_{m}\right)^{2}-\mathcal{C}_{2}\left(\hat{\varphi}_{r q}\right)^{2}$

For $\mathcal{C}_{1}>0$ and $\mathcal{C}_{2}>0$, then:

$\hat{\varphi}_{r}=\hat{\varphi}_{r d}+j \hat{\varphi}_{r q} \rightarrow \varphi_{m}$

Under this condition, $\hat{\varphi}_{r q}=-\varphi_{m} \sin \tilde{\theta}$. Wheı $\hat{\theta}_{2}$ is approximated with $\theta \quad\left(\hat{\theta}_{2} \equiv \theta\right)$, using (63) to test the adaptation condition.

$\frac{d \widetilde{\omega}}{d t}=\sigma \varphi_{m} \hat{\varphi}_{r q}$

Finally, if $\omega$ is supposed constant, -quation (66) becomes:

$\frac{d \widehat{\omega}_{2}}{d t}=\sigma \varphi_{m} \hat{\varphi}_{r q}$

\subsubsection{Design of ...e $\mathrm{Pl}$ - egulator}

In practice if the neer variation is slow compared to the adaptation time constant, which

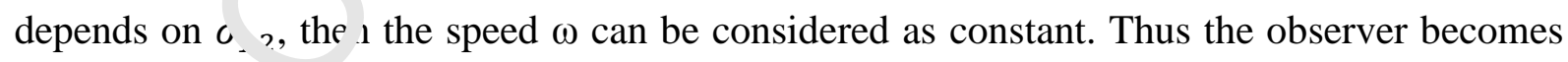
[34]: 


$$
\left\{\begin{array}{l}
\hat{\varphi}_{r}=\hat{\varphi}_{s}-L i_{s} \\
\hat{\varphi}_{s}=\int_{0}^{t}\left(v_{s}+R_{s} i_{s}-\mathcal{C}_{1}\left(\hat{\varphi}_{r d}-\varphi_{m}\right)-j \mathcal{C}_{2} \hat{\varphi}_{r q}-j \widehat{\omega}_{2} \hat{\varphi}_{s}\right) d t \\
\widehat{\omega}_{2}=\sigma_{1} \hat{\varphi}_{r q}+\sigma_{2} \int_{0}^{t} \hat{\varphi}_{r q} d t \\
\hat{\theta}_{2}=\int_{0}^{t} \widehat{\omega}_{2} d t
\end{array}\right.
$$

Where $\sigma_{1}$ and $\sigma_{2}$ are gains of the PI regulator, $\mathcal{C}_{1}$ and $\mathcal{C}_{2}$ are posit ve $c$ snsunts.

\subsubsection{Design of the Fuzzy Controller}

Classical PI controllers are very sensitive to internal $f$ aumeıs variations and external disturbances. Fuzzy logic controller (FLC), on the other hand, h . ve attractive features such as robustness and hence overcomes these problems.

The FLC includes four basic components: fuz : aumivin, fuzzy rule base, fuzzy inference engine and defuzzification [46].

For this system, the quadrature flux $\hat{\varphi}_{r q}$ and to thange $d \hat{\varphi}_{r q}$ are the inputs of this FLC. The output represent the estimated speed, where $\iota_{\text {. }}$ flux variation $d \hat{\varphi}_{r q}$ is defined by :

$$
d \hat{\varphi}_{r q}=\left(\hat{\varphi}_{r q}(t+1)-\hat{\varphi}_{r q}(t)\right) / T
$$

Where $T$ is the sampling period

Three membership function with trangular and trapezoidal symmetrical equidistant shaps are defined for each inpr \& v r riable with the universe of discourses $[-2,2]$ for $\hat{\varphi}_{r q}$ and $[-1.2$, 1.2] for $d \hat{\varphi}_{r q}$ respect velv. ' 1 t.e output has nine triangular membership functions defined in the universe of dis _urse : 20,20]. The membership functions for the inputs and outputs are shown in Figure 4. The uzzy subset of linguistic variables are labelled as Negative Big (NB), Negative Sm 'll (N`), Negative (N), Negative Medium (NM), Zero (Z), Positive Medium (PM), Pos` 'iv $f(r)$, Positive Small (PS), Positive Big (PB). All the fuzzy inference rules are summarized in Table 1, where the AND fuzzy operation intersection is applied between the inputs. The max Mamdani method was used in the inference mechanism. The center of 
gravity was employed in the defuzzification, to transform the fuzzy set resulting from the rules aggregation into a control quantity, which represents the estimated speed:

$$
\widehat{\omega}_{2}(k)=\frac{\sum_{i=1}^{n} U_{i} \mu\left[U_{i}\right]}{\sum_{i=1}^{n}\left[U_{i}\right]}
$$

Where $\mu\left[U_{i}\right], n$ and $U_{i}$ is the degree of membership value, the nur..'er $v_{-}^{-}$the fuzzy rules (equal to 9 in this case) and the center of gravity of the $i^{\text {th }}$ fuzz $\mathrm{se}^{t}$ respectively $[44,45]$. $k_{1}, k_{2}$ and $k_{3}$ are adaptation gains which determine th $\leftarrow$ tra: sient and steady-state characteristics of the FLC controller [48].

The direct reference current at low speeds is changed ai. ${ }^{\mathcal{T}}$ takes $\_$non-zero value, which can be determined from [34] as:

$i_{r e f}^{d}=i_{0}^{d} e^{-\widehat{\omega} / \omega_{0}}$

Where $i_{0}^{d}$ is the maximum current and $\omega_{0}$ is $\mathrm{t}_{\mathrm{L}} \cdot \mathrm{l}, \mathrm{W}$ speed. These parameters $i_{0}^{d}$ and $\omega_{0}$ are determined from [34].

\section{Switching Algorithm}

The transition between the observe ; is act eved using two weighting coefficients $\alpha$ and $\eta$, as shown in Figure 5. Where $\alpha$ rf pres unts the weighting of the speed obtained at low regime, while $\eta$ is the weighting $\mathrm{o}^{\prime}$ the speed obtained from HOSMO. $\alpha$ Fully prevails below a threshold speed $\Omega_{1}=9 \mathrm{ra}{ }^{\prime} / \mathrm{s}$, however, $\eta$ dominates above a threshold speed $\Omega_{2}=$ $25 \mathrm{rad} / \mathrm{s}$. The transit; $\mathrm{n}$ is ac ivated for a speed $\Omega_{1}<\omega<\Omega_{2}$ where a common information are in this region.

It is important to 'nte $t^{t}$ at the effectiveness of this method depends essentially on the choice of the transi ion rę on. This transition zone can be determined from the maximum speed above wh, h $\mathrm{t}$ « iow speed observer performance deteriorates, and the minimum speed below which the HOSMO performance deteriorates [21, 23]. The classical algorithm [3, 23] imposes a single speed in each region. However, in case of divergence of one of the observer, the system remains in a poor performance until the intervention of the second observer, then 
control is completely lost if no reference change is applied. The modified switching strategy overcomes these drawbacks and improves the performance of the system. The modified algorithm is illustrated in Figure 6.

\section{Simulation Results}

The simulation is performed in MATLAB/Simulink. To demons. "ate the effectiveness of the proposed approach, the following wind profile is used:

$V_{w}=10+0.2 \sin 0.1047 t+2 \sin 0.2665 t+\sin 1.2930++0^{2} \sin 3.664 t$

The low wind speed operation starts from $t=0$ to $3.5 \mathrm{~s}$, ith a value of $0.1 V_{w}$, the transition is applied during the acceleration. From $t=4$ to $1 \mathrm{C} c$ th $~ w i d$ nominal speed takes the value of $V_{w}$, then decreases back to the first value betwec. $t=11 \mathrm{~s}$ and $t=15.5 \mathrm{~s}$. Finally, the wind speed changes its value from 0.1 to $0.5 V_{w}$ for $\mathrm{L}$ e iest of the time. The perturbation changes under different operation conditions and its crervative is limited even if the perturbation is unbounded or varies over time. A large disı bance with low HOSMO coefficients can be lead to instability of system, sr all dis urbance with HOSMO coefficients can cause chattering. For this reason, The ate ${ }^{\prime} \mathrm{mit}^{+}$of the perturbation change is given by $\varrho_{\alpha . \beta}=4$, and the HOSMO coefficients i. chosen to achieve a compromise between the robustness, convergence rate and t'e ize of control action. Based on the basis of the following parameters :

$\varepsilon=1, \varrho_{1,2}=4, \Gamma=0 . c^{2}$. Where the HOSMO coefficients are calculated from (23), (24), (38) and (39) .

$\mathcal{K}_{1}=0.5, \mathcal{K}_{2}-{ }^{r}, \mathcal{K}_{3}=8$ and $\mathcal{K}_{4}=3$

(switching : equency is $15 \mathrm{kHz}$ ). The gains of the speed PI controller are set to: $k p_{w}=0.2$ and $k i_{w}=0.4 . k p_{i}=4$ And $k i_{i}=570$. For the observer at low speed: $\sigma_{1}=100$ and 
$\sigma_{2}=10, \mathcal{C}_{1}=250$ and $\mathcal{C}_{2}=5$. Table 2 give the various parameters values of the machine and turbine used in the simulation model.

The control associated with these observers guarantee a good transie' $\mathrm{c}$ a. $d$ steady-state performance in the nominal, medium and low regime simultaneously $r$ th $f$ transition region. From the first comparison, the FLC demonstrated better performance than PI control as shown in Figure 7 (right panel) and Figure 7 (left panel) respect ${ }^{-1} \%$. Figure 7 a shows that the estimated generator speed follows reasonably well its referer ${ }^{2}$ with good response characteristics and accuracy. There is no discontinuity be wef . . vo speed regions, and there is almost no influence and no performance degradation c. ntırming the effectiveness of the proposed observer structure and control scheme. $k_{\text {. }}$ reov., no chattering appeared at high speeds. Figure $7 \mathrm{~b}$ indicates two separate speed reglı $` s$ where the low speeds are reserved only for the second observer and after $25 \mathrm{rad} / \mathrm{s}$ the $1 \mathrm{r}$ SMO is activated. It can also be observed that the second observer during the high-spe $d$ ıegion is about zero. Figure $7 \mathrm{~d}$ shows the estimated rotor position, which can be clearly seen to track exactly the measured position. During this speed change, the elec.' 'mech nical torque undergoes a sudden peak which then quickly vanishes as shown in F gur - 8f Note that these peaks are not expected to cause any damage to the machine. Thr sults show that the FLC performance is superior to that of PI control and the peaks ar ev $\mathrm{n}$ reduced. The current $i_{q}$ has the same form as the torque, and the decoupling achie ed was successfully by maintaining $i_{d}=0$. The current $i_{d}$ takes two different values $0 \div$ at hly: speed and $5 A$ at low speed as depicted in Figure 7e. The optimal values of $C_{p}$ and $\wedge^{-h n} \mathrm{n}$ in Figure $8 \mathrm{~g}$ clearly illustrate the performance of the MPPT, where Figure 7c shc vs th ${ }^{\circ}$ current components along $\alpha$ and $\beta$ axes which have a sinusoidal shape. The three-p s se currents $i_{a b c}$ is shown in Figure 7h. Finally, it can be seen that the signal with FLC improve the signal quality at transition region. 
Figure 8A shows that the HOSMO loses its control in its region but, with the proposed algorithm, the observer at low speed continue to operate until HOSMO is activated again (Figure 8A left side). However, with the classical algorithm, HOSMO is 'elt ${ }^{+a d}$ despite the poor performance.

The transient speed response is better with the proposed new approach (-igure 8B, left side) than the original approach (Figure 8B, right side). In the (Figure ${ }^{\mathrm{C}}$. left side), the proposed structure favors HOSMO even though it is not supposed o ope tte here. However, the classical structure maintains the second observer despitf the iterioration of the response. After the comparison it can be observed that the two algos ${ }^{+} \mathrm{hms}$ perform the same operation, but with the original algorithm, each observer is spon. ${ }^{2}$ le to its region, this can cause unacceptable excursions that can cause damage th machine. On the contrary, the proposed algorithm is able to balance between the two c ' srvers and does not let the loss of control affect the performance of the machine. Th. purformance of the proposed algorithm is acceptable in all phases.

Finally, Table 3 shows the compar: $n$ am ng five methods.As a summary, the advantages of the proposed method are the fo low ng: 1) Guaranteed at wide speed range; 2) Only for Non salient pol NSPPMSM; 3) -'nnfirmed at wide speed range; 4) take into consideration the losing control; and 5) the pos tion error in the transition zone is 0.06 (rad).

\section{Conclusion}

In this paper, a new hyc ${ }^{-i}{ }_{1}$ observer is proposed to estimate the position and velocity of a

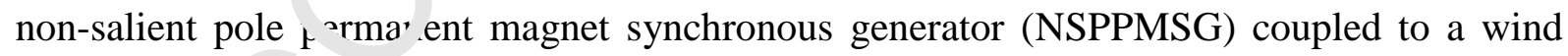
turbine that ( nerates over a wide speed range. The proposed hybrid observer consists of two observers our mi in cascade using a modified weighting coefficients method. The low-speed observer is specially adapted to this type of machine and is able to determine the mechanical effect caused by the quadrature axis rotor flux. The second observer, high order sliding mode observer operates at medium and nominal wind speed, which is designed based on a modified 
structure of the super twisting algorithm. The second observer gains are designed such us to limit the impact of a linearly growing perturbation. The stability of each observer is guaranteed by Lyapunov stability analysis. The simulations results at low 'pec.' show that the fuzzy logic based regulator gives better performance than the $r$ assi al PI regulator. Moreover, another comparison confirms the effectiveness of the roposed switching algorithm, and these results are satisfactory in the nominal, mea $r$ and low case scenarios and are the same at the transition region, where its show 10 inflı ence under wind speed variations.

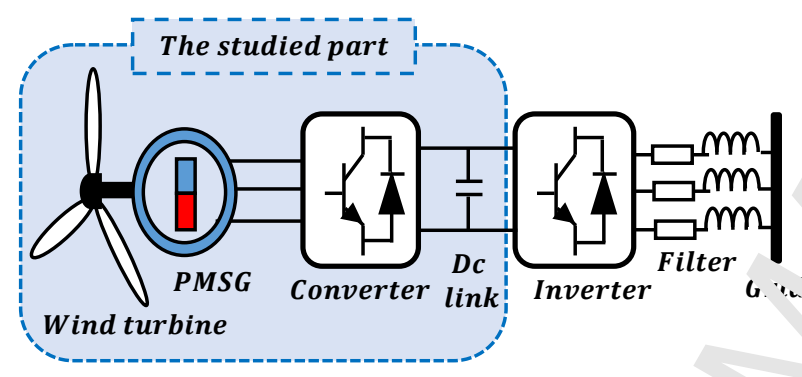

Fig. 1. Configuration of a $P M S G$ wind turbine

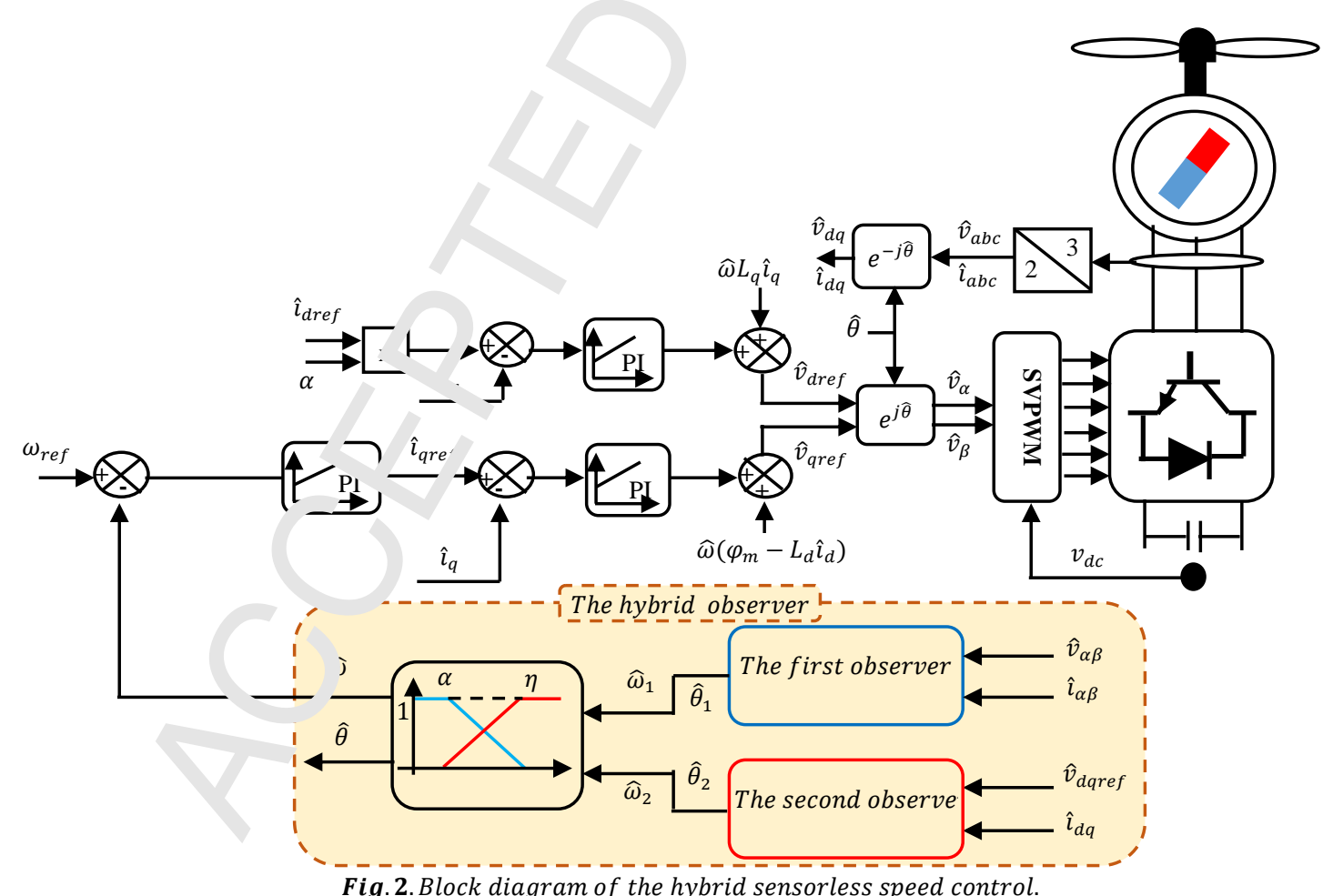




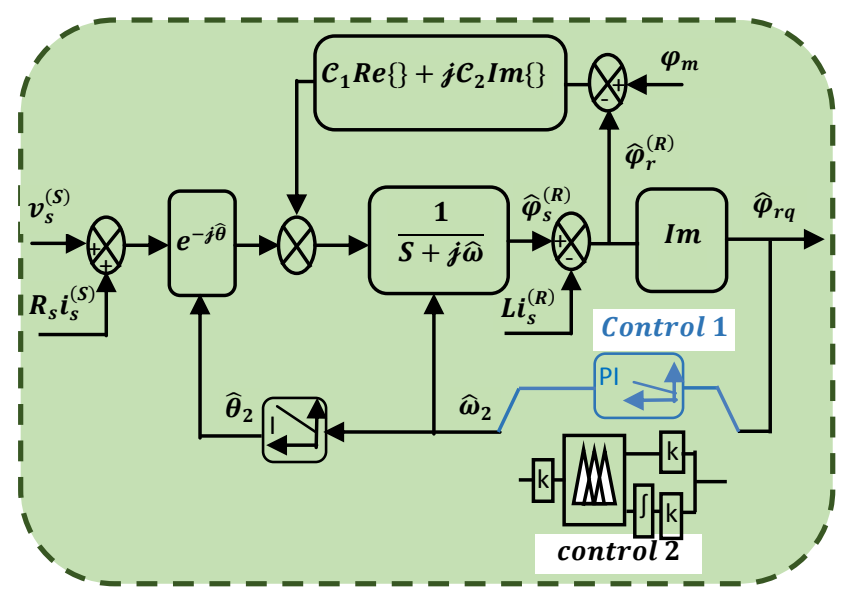

Fig. $\overline{3} \cdot \overline{\text { Second }}$ observer
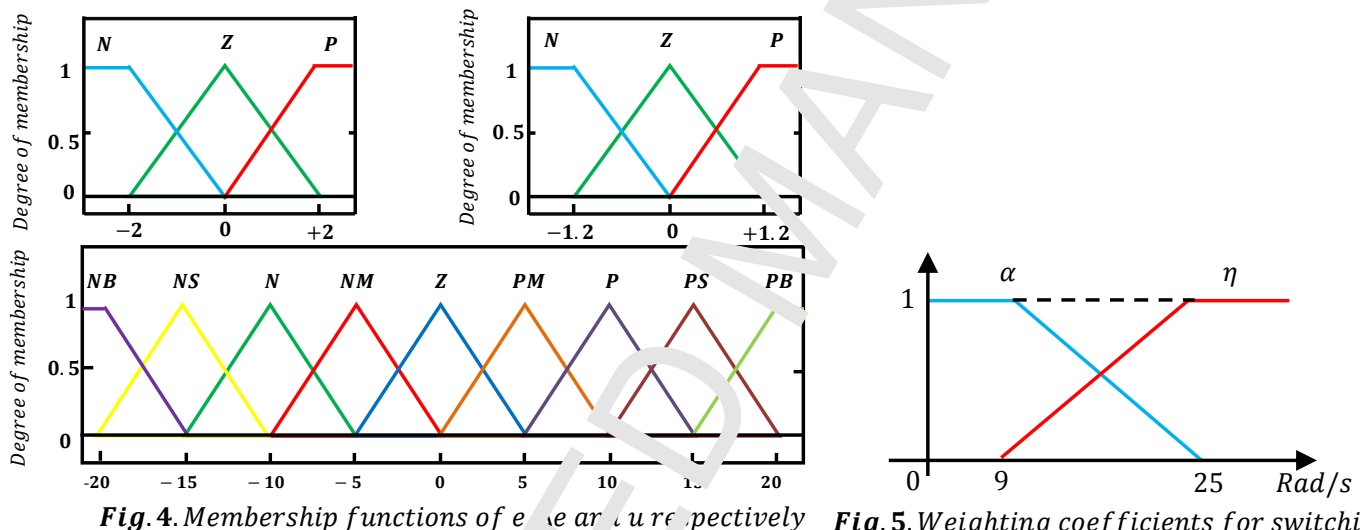

Fig. 4. Membership functions of e ve ar a re pectively Fig. 5. Weighting coefficients for switching algorithm 


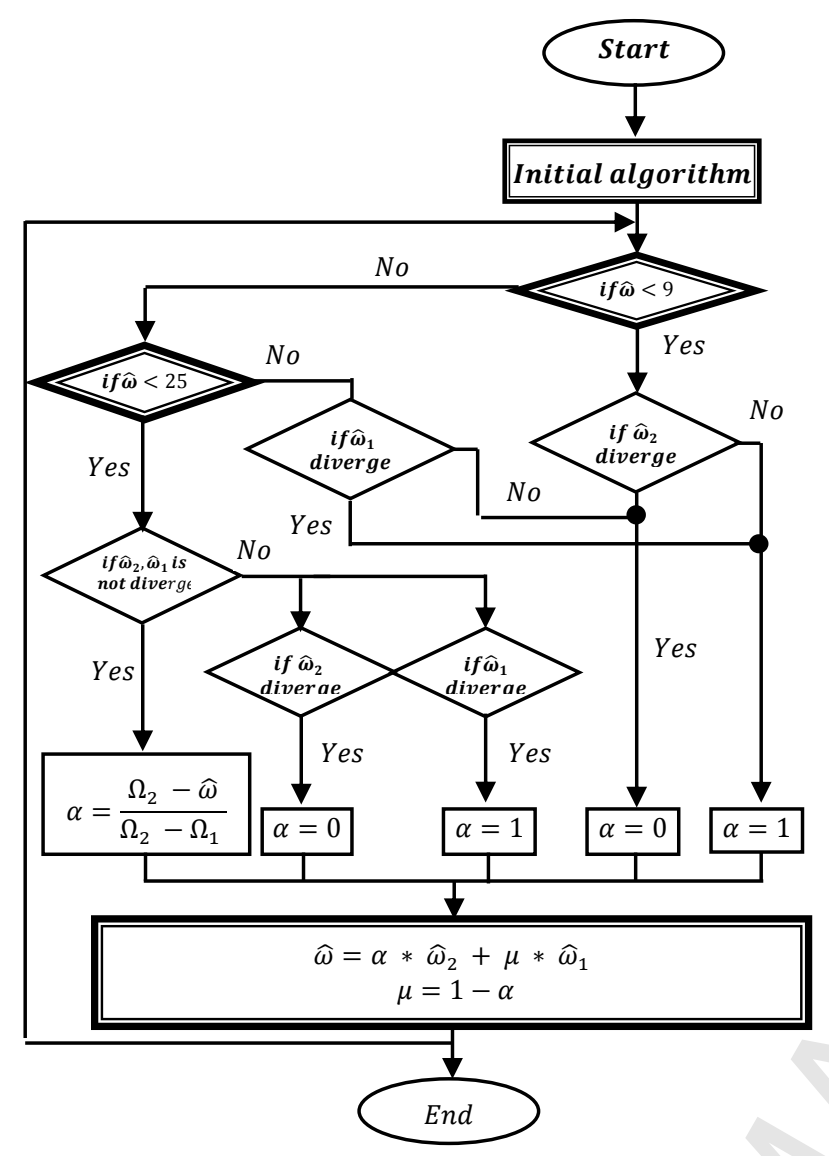

Fig. 6. Proposed Switching Algorithm 

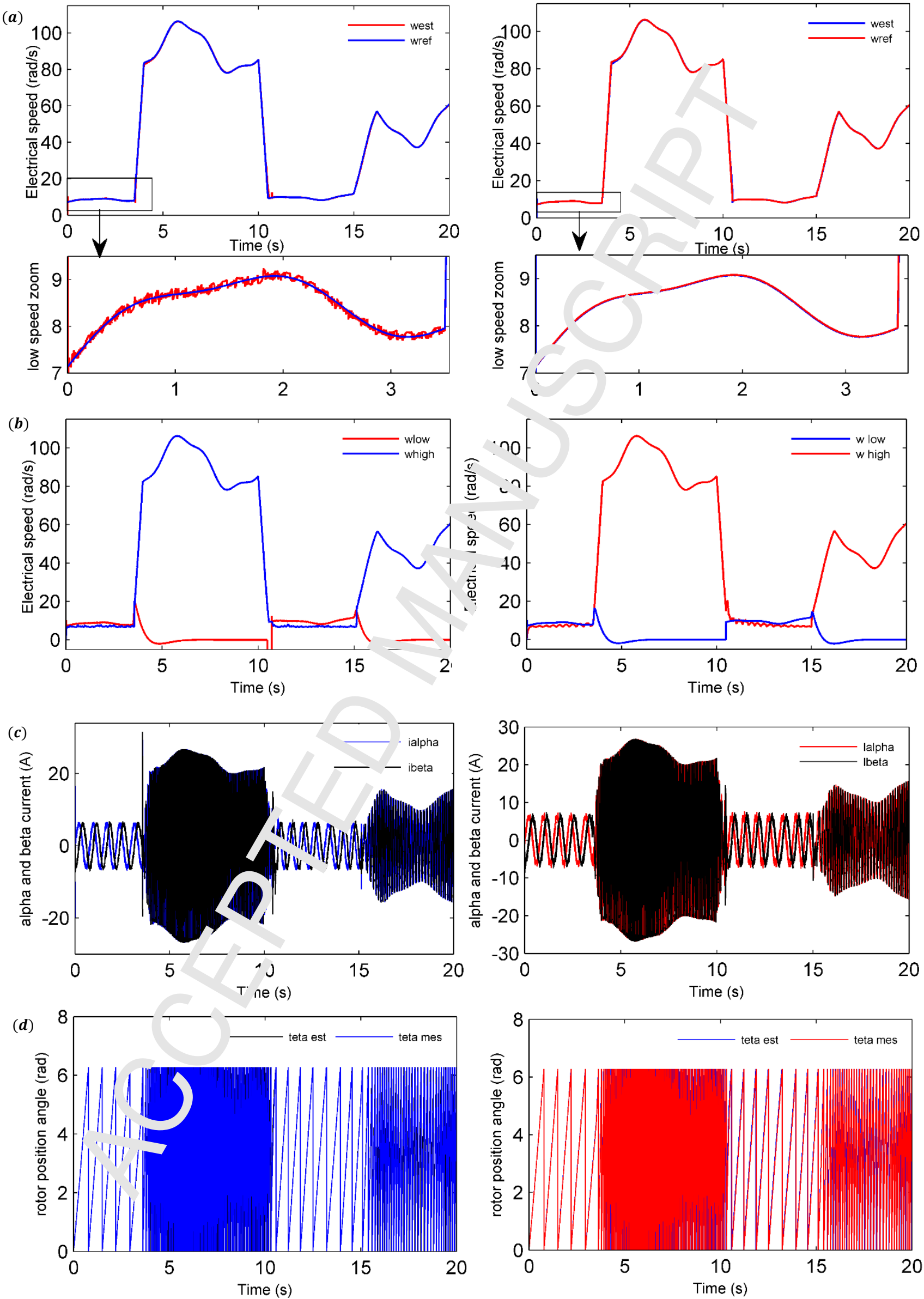

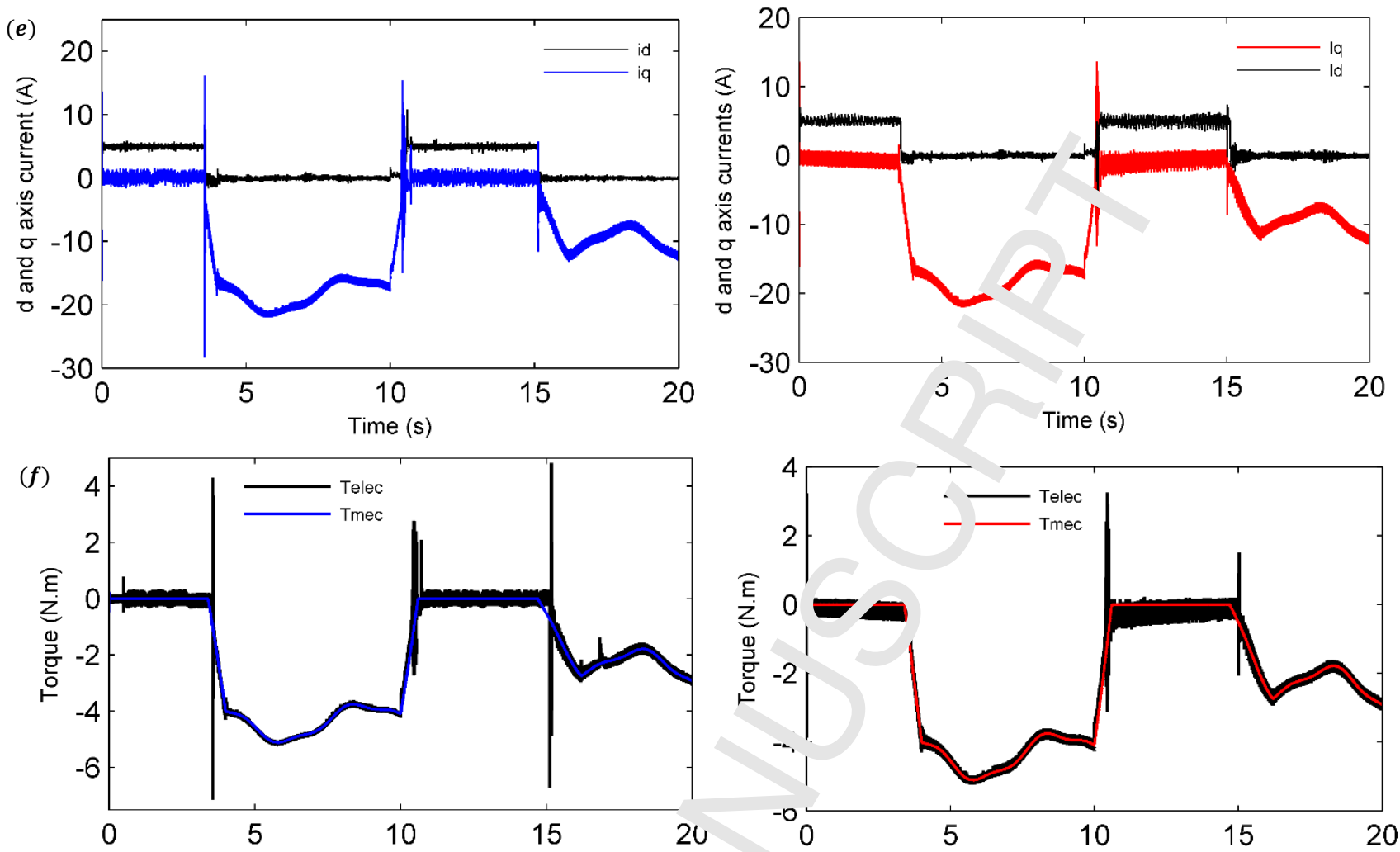

(g)
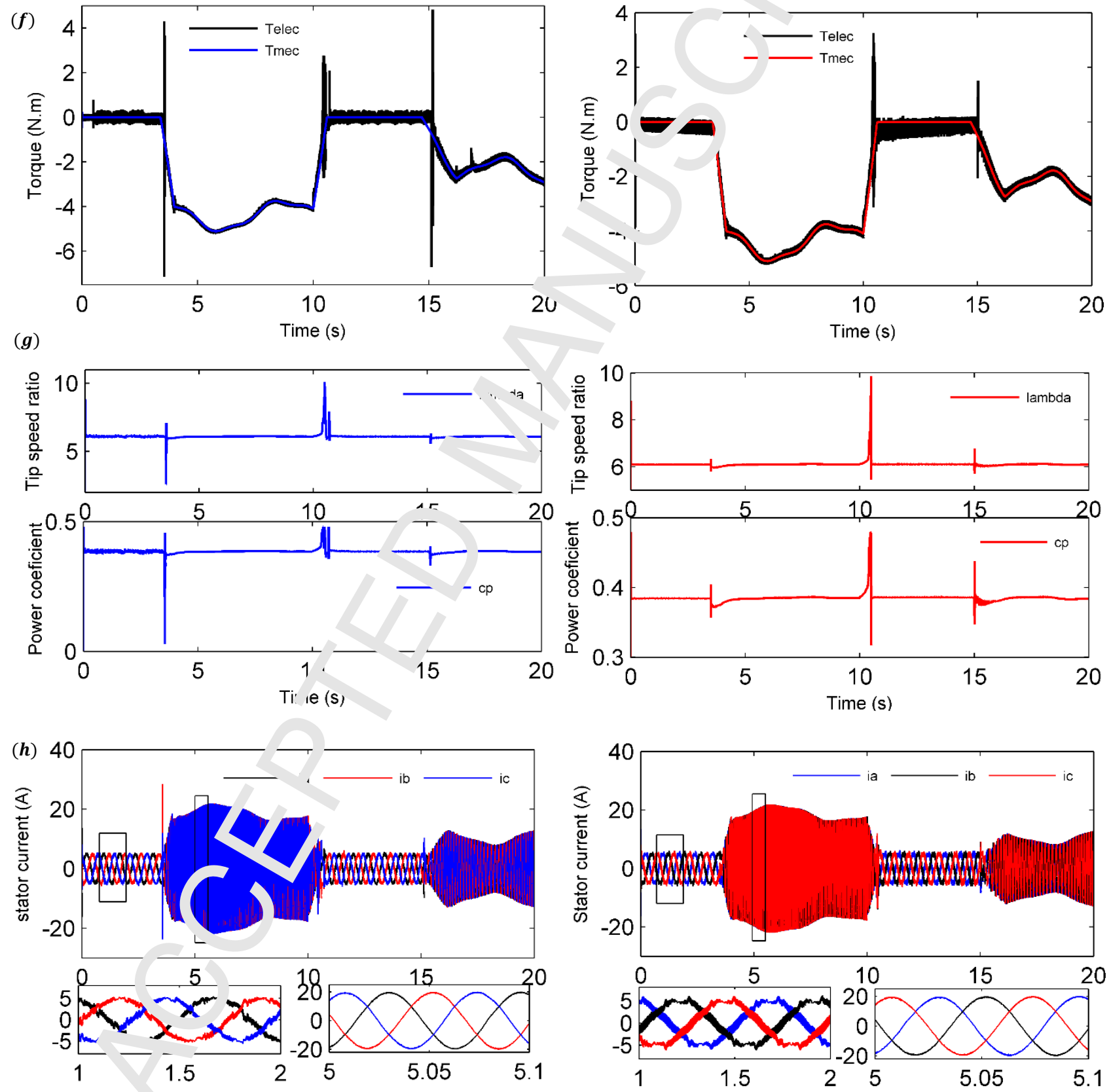

Fig. 7. (left side) PI controller; (right side) Fuzzy controller. 
(A)
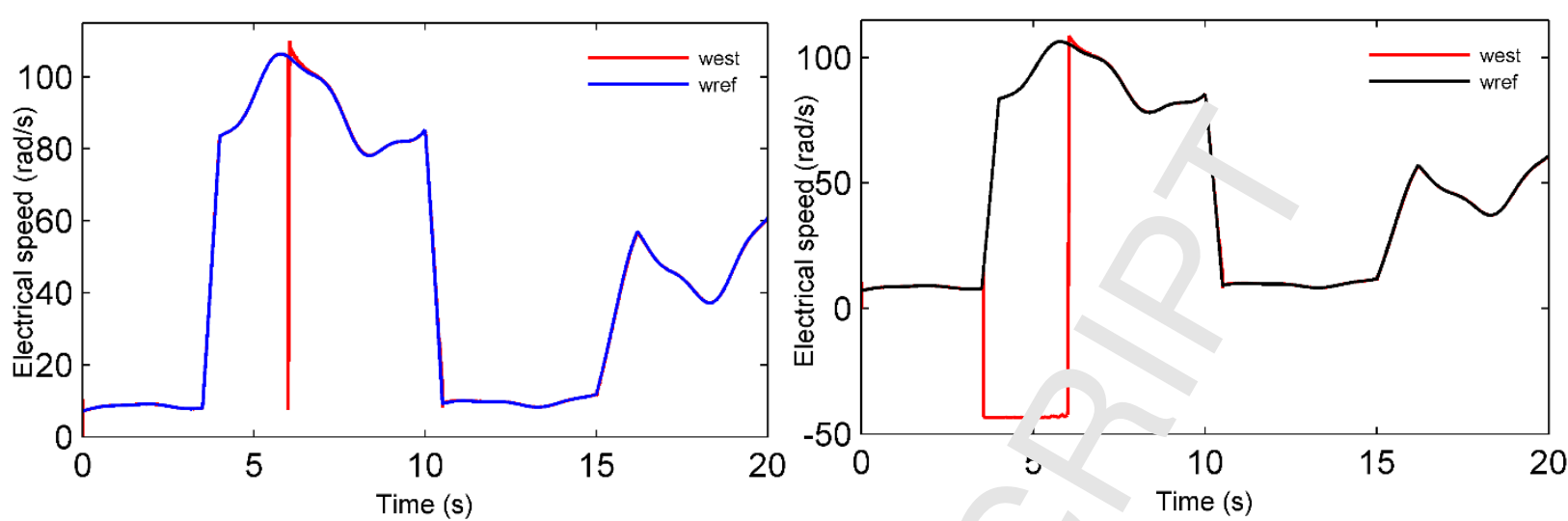

(B)
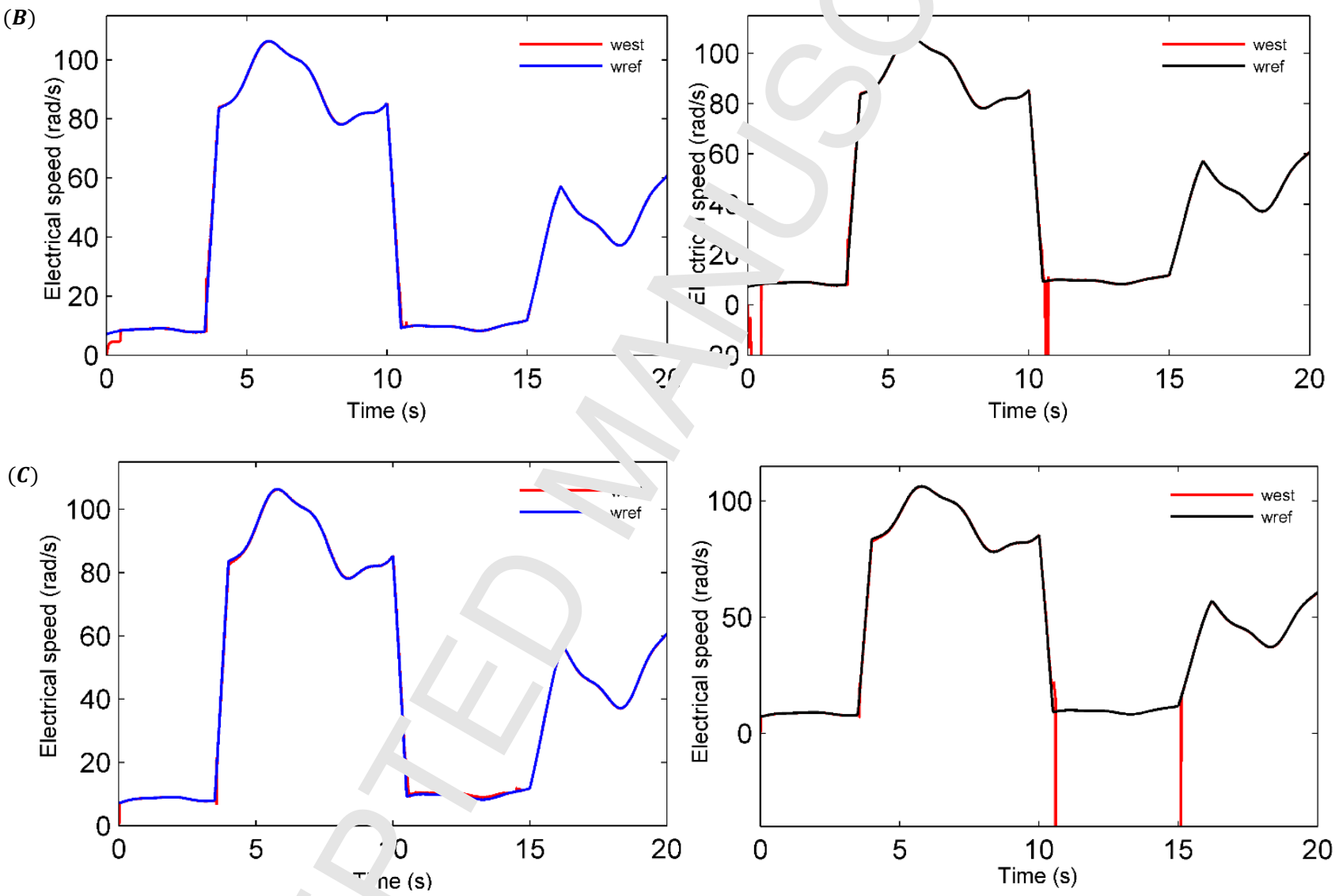

F'g. 8. (,ight side) traditional algorithm (left side) proposed algorithm.

Table 1 - Fuzzy co trol rul

\begin{tabular}{ccccc}
\hline \multirow{2}{*}{$\mathbf{d u}$} & & \multicolumn{3}{c}{$\boldsymbol{d} \widehat{\boldsymbol{\varphi}}_{r \boldsymbol{q}}$} \\
\cline { 3 - 5 } & & $\mathbf{N}$ & $\mathbf{Z}$ & $\mathbf{P}$ \\
\hline \multirow{3}{*}{$\widehat{\boldsymbol{\varphi}}_{r q}$} & $\mathbf{N}$ & $\mathrm{NB}$ & $\mathrm{NS}$ & $\mathrm{P}$ \\
& $\mathbf{Z}$ & $\mathrm{NM}$ & $\mathrm{Z}$ & $\mathrm{PM}$ \\
& $\mathbf{P}$ & $\mathrm{N}$ & $\mathrm{PS}$ & $\mathrm{PB}$ \\
\hline
\end{tabular}


Table 2 - A machine parameters.

\begin{tabular}{lcc}
\hline Parameters & Values & Units \\
\hline Stator Resistance Rs & 0.57 & {$[\Omega]$} \\
Stator Inductance L & 0.004 & {$[\mathrm{H}]$} \\
Back EMF constant $K_{e}$ & 0.078 & {$[\mathrm{Vs} / \mathrm{rad}]$} \\
viscous friction $f_{v}$ & 0.004 & {$[\mathrm{Nm} \cdot \mathrm{s} / \mathrm{rad}]$} \\
Rotor flux $\phi_{m}$ & 0.064 & {$[\mathrm{wb}]$} \\
Moment of Inertie $\mathcal{J}$ & 0.002 & {$\left[{\left.\mathrm{Kg} . \mathrm{m}^{-2}\right]}\right.$} \\
Number of pole paires np & 2 & \\
\hline
\end{tabular}

Table 3: Comparison between different approaches.

\begin{tabular}{|c|c|c|c|c|c|}
\hline \multirow{2}{*}{ Performance criteria } & \multicolumn{3}{|r|}{ comparison } & \multirow[b]{2}{*}{ [22] } & \multirow[b]{2}{*}{ [23] } \\
\hline & Proposed approach & [3] & [20] & & \\
\hline robustness & $\begin{array}{c}\text { Guaranteed at wide } \\
\text { speed range }\end{array}$ & $\begin{array}{c}\text { robust at high } \\
\text { speed }\end{array}$ & $\begin{array}{c}\text { robust ainst } \\
\text { parameter } \\
\text { vari ition at high } \\
\text { spu }\end{array}$ & $\begin{array}{l}\text { obust against } \\
\text { parameter } \\
\text { variation at high } \\
\text { speed }\end{array}$ & $\begin{array}{c}\text { robust against } \\
\text { parameter } \\
\text { variation at high } \\
\text { speed }\end{array}$ \\
\hline simplicity & simple structure & simple structure & simple. "icture & $\begin{array}{l}\text { complicated } \\
\text { structure }\end{array}$ & simple structure \\
\hline limitations & $\begin{array}{c}\text { Only for Non salient } \\
\text { pol NSPPMSM }\end{array}$ & $\begin{array}{l}\text { For machine with } \\
\qquad L_{d} \neq L_{q}\end{array}$ & $\begin{array}{c}1 \quad \text { Surface } \\
\text { Monnted PMSM }\end{array}$ & $\begin{array}{l}\text { Only For interior } \\
\text { PMSM }\end{array}$ & $\begin{array}{c}\text { For Surface } \\
\text { Mounted PMSM }\end{array}$ \\
\hline stability & $\begin{array}{l}\text { Confirmed at wide } \\
\text { speed range }\end{array}$ & $\begin{array}{c}\text { Not confirmed at } \\
\text { low spe }\end{array}$ & Not confirmed & Not confirmed & $\begin{array}{c}\text { Not confirmed at } \\
\text { low speed }\end{array}$ \\
\hline Losing control & $\begin{array}{c}\text { take into } \\
\text { consideration }\end{array}$ & $\begin{array}{l}\text { No take into } \\
\text { cor. }\end{array}$ & $\begin{array}{l}\text { No take into } \\
\text { consideration }\end{array}$ & $\begin{array}{l}\text { No take into } \\
\text { consideration }\end{array}$ & $\begin{array}{l}\text { No take into } \\
\text { consideration }\end{array}$ \\
\hline $\begin{array}{l}\text { Position error in the } \\
\text { transition zone (rad) }\end{array}$ & 0.06 & 0.12 & 0.08 & 0.07 & 0.1 \\
\hline
\end{tabular}

\section{REFERENCES}

[1] Shengquan Li, Kezhao Zr ang, Jua.. Li, Chao Liu, On the rejection of internal and external disturbances in a wind $\ldots$ rgyConversion system with direct-driven PMSG, ISA Trans. 61, (2016), 95-103.

[2] R. Fantino, J. Soı na C. Busada, Non-lineair observer-based control for PMSG wind turbine, Energ, $113($ ( J16) 248-257.

[3] I. Omran , E. Et. „n, W. Dib, O. Bachelier, Modeling and simulation of soft sensor design for rea ra- sneed and position estimation of PMSM, ISA Trans. 57 (2015) 329-339.

[4] S. Bologn، ni, S. Calligaro, R. Petrella, Design Issues and Estimation Errors Analysis of Back-EMF-Based Position and Speed Observer for SPM Synchronous Motors, IEEE 
Journal of Emerging and Selected Topics in Power Electronics, 2 (June (2) (2014)) 159170.

[5] D. Xu, S. Zhang, J. Liu, Very-low speed control of PMSM based on E At - ctimation with closed loop optimized parameters, ISA Trans. 52 (2013) 835-843.

[6] Y. Shi, K. Sun, L. Huang, Y. Li, Online identification of permanent 1 . ngnet flux based on extended kalman filter for ipmsm drive with position sensorles $r$ sntrol, IEEE Trans. Ind. Electron. 59 (November (11)) (2012), 4169-4178.

[7] Z. Q. Guo, S. K. Panda, A Novel Design of Rotor Po ‘a n Estimator for Sensorless Control of SPMSM Operating at Medium and High $\supset_{1}$ seas, IEEE PEDS 2015, Sydney, Australia, june 2015,pp 1080-1085.

[8] M. Pacas, Sensorless drives in industrial appı ^tions, IEEE Ind. Electron. Mag. 5 (june (2)) (2011), 16-23.

[9] S. K. Kommuri, M. Defoort, H. R. Kariı i, 1s. C. Veluvolu, A Robust Observer-Based Sensor Fault-Tolerant Control for PMs $\triangle_{\wedge}$ in Electric Vehicles, IEEE Trans. Ind. Electron.63 (December (12)) (2C 6), 76 ’1- 7681.

[10] Si Yi Chen, Ying Luo, ' our,uo Pi, PMSM sensorless control with separate control strategies and smooth swi ,

[11] H. Kim, J. Son, J. Lee A high-speed sliding-mode observer for the sensorless speed control of a PMSM IEFE i ans. Ind. Electron. 58 (September (9)) (2011), 4069-4077.

[12] Vasilios C. Ilijua . Chattering reduction applied in pmsm sensorless control using second order si ting aode observer, in Proc. 9th Int. Conf. Compat. Power Electron, June 2015, pp.2 ${ }^{10-245}$

[13] M. L'za', „. de Leon, N. Gonzalez, A. Glumineau, Observer controller scheme using high order sliding mode techniques for sensorless speed control of permanent magnet synchronous motor, in Proc. 49th IEEE Conf. Decision Control, Dec 2010, pp. 4012-4017. 
[14] J.A. Moreno, "A linear framework for the robust stability analysis of a generalized super twisting algorithm, " 6th International Conference on Electrical Engineering, Computing Science and Automatic Control, Toluca, Mexico, 2009, pp. 12- 17.

[15] L. Zhao, J. Huang, H. Liu, B. Li, W. Kong, Second-order slidir \& m de observer with online parameter identification for sensorless induction motor drive. IEEE Trans. Ind. Electron, 61(October (10)) (2014), 5280-5289.

[16] J. A. Moreno, M. Osorio, Strict Lyapunov functions $f(r$ the $s$ per-twisting algorithm, IEEE Trans. Automat. Control, 57 (April (4)) (2012), 1r 35- v'0.

[17] T. Gonzalez, J. Moreno, L. Fridman, Variable gain sı..er-twisting sliding mode control, IEEE Trans. Automat. Control, 57 (August (8)) (2^12), 2: J0-2105.

[18] J. Lara, A. Chandra, Performance Investiga: in of Two Novel HSFSI Demodulation Algorithms for Encoderless FOC of PMSMs , it anded for EV Propulsion, IEEE Trans. Ind. electron, 65 (July (2)) (2017), 1074 - 1083.

[19]Z. Q. Zhu, A.H. Almarhoon, P. L. Xu, Inıroved Rotor Position Estimation Accuracy by Rotating Carrier Signal Injectior Utilizi g Zero-Sequence Carrier Voltage for Dual ThreePhase PMSM, IEEE Trans. Ir d. e ectron, 64 (May (6)) (2016), 4454 - 4462.

[20] C. Silva, G.M. Asher, nd M. Sumner, Hybrid rotor position observer for wide speed range sensorless PM not $\mathrm{r}$ drives including zero speed, IEEE Trans. Ind. Electron. 53 (April (2)) (2006), $-73-3,3$.

[21] S. Sayeef, G Foo, Y. F. Rahman, Rotor position and speed estimation of a variable structure direc. tora e-controlled IPM synchronous motor drive at very low speeds including : tandsti l, IEEE Trans. Ind. Electron., 57 (November (11)) (2010), 3715-3723.

[22] G. W ng 1.. Yang, D. Xu, DSP-Based Control of Sensorless IPMSM Drives for WideSpeed-Range Operation, IEEE trans. Ind. electron, vol. 60 (February (2)) (2013), 720-727.

[23] Z Ma, J Gao, R. Kennel, FPGA Implementation of a Hybrid Sensorless Control of SMPMSM in the Whole Speed Range, IEEE trans. Ind. Inf., 9 (august (3)) ( 2013) 1253 - 
1261.

[24] W. Jianmin, Performance of pulsating high-frequency current injection based sensorless

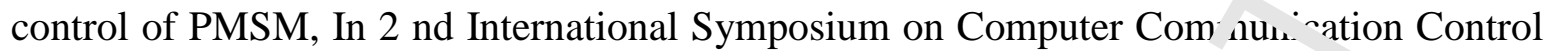
and Automation (ISCCCA), 2013.

[25] J. M. Liu and Z. Q. Zhu, A new sensorless control strategy by higı frequency pulsating signal injection into stationary reference frame, In IEEE Inte. ${ }^{\wedge}$ onal Electric Machines and Drives Conference (IEMDC), 2013, pp 505-512.

[26] M. Boussak, Implementation and experimental inves iga $^{{ }^{*}}{ }_{*}$ of sensorless speed control with initial rotor position estimation for interior perm nent magnet synchronous motor

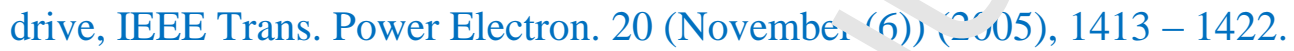

[27] H. Kim, K. K. Huh, R. D. Lorenz, and T. Iv Jahns, A novel method for initial rotor position estimation for IPM synchronous i 7 nine drives, IEEE Trans. Ind. Appl, 40 (October (5)) (2004), $1369-1378$.

[28] M. Schroedl E. Robeischl, Optimized $\Perp$ iFORM-measurement sequence for sensorless PM synchronous motor drives ith res sect to minimum current distortion. IEEE Trans. Ind. Appl. 40 (April (2)) $(20 \odot+), \digamma 91>598$.

[29] Y.D. Yoon, S.K. Sul, S Morimoto and K. Ide, High bandwidth sensorless algorithm for AC machines based $r$ a s uare-wave type voltage injection, IEEE Trans. Ind. Appl, 47 (June (3)) (2011), 1,61-13, ).

[30] A. Madani, J. D Bå ${ }^{\prime}{ }_{\mathfrak{i}}$, F. Colamartino, C. Marchand, Reduction of torque pulsations by inductance ha noniss identification of a permanent-magnet synchronous machine, Proceedin $_{\varepsilon} s$ of th 4 th IEEE Conference on Control Applications, 1995, pp. 787-792.

[31] G. F( ?, 1. ₹. Rahman, Sensorless sliding-mode MTPA control of an IPM synchronous motor drive using a sliding-mode observer and HF signal injection, IEEE Trans. Ind. Electron., 57 (April (4)) (2010), 1270-1278. 
[32] G.-D. Andreescu, C. Pitic, F. Blaabjerg, I. Boldea, Combined flux observer with signal injection enhancement for wide speed range sensorless direct torque control of ipmsm drives, IEEE Trans. Energy Convers, 23 (June (2)) (2008), 393 -402.

[33] S. Bolognani, A. Faggion, E. Fornasiero, L. Sgarbossa, Full speer ran „e sensorless IPM motors drives, In: International conference on electrical marhint ICEM, Marseille, France; 2012.

[34] H. Rasmussen, Sensorless speed control including $\mathrm{z}$ ro $\operatorname{sp}$ ed of non salient PM synchronous machines, Bull. Pol. Ac. Tech. 54(3) 2006

[35] H. Dong, C. Zhang, D. Wang, S. Xu, J. Qiu, Dynan` - cnaracteristics of gear box with PGT for wind turbine, The 7th Internation. ${ }^{\prime}$ c_...erence on Sustainable Energy Information Technology, Procedia Computer Sc. nce 109C (2017), pp 801-808.

[36] M. Ma, L. Shao, X. Liu, Coordinated contrc' ' $\dot{i}$ micro-grid based on distributed moving horizon control, ISA Trans. 76 (2018), 216-?23.

[37] D. Zaltni, M. N. Abdelkrim, Robust speed and position observer using HOSM for sensor-less SPMSM control, in Jroceec ngs of the 7th International Multi-Conference on Systems, Signals and Device , ( $\left.S^{\top}, D^{\prime} ! 0\right)$, June 2010, pp. 1-6.

[38] A. Chalanga, K. St , ^m, L. M. Fridman, B. Bandyopadhyay, J. A. Moreno, Implementation of $\mathrm{Sr}$ per Twisting Control: Super-Twisting and Higher Order SlidingMode Observer-Ba ed App. Jaches, IEEE Trans. Ind. Electron. 63 (6) (2016), 3677-685.

[39] V. Utkin, Dincuss $\cdots$ Aspects of High Order Sliding Mode Control, IEEE Trans. Automat. Conı ר1, 61 ,March (3)) (2016), 829 - 833.

[40] S. K. K rmmur, C. K. Veluvolu, M. Defoort, Y. C. Soh3, Higher-Order Sliding Mode

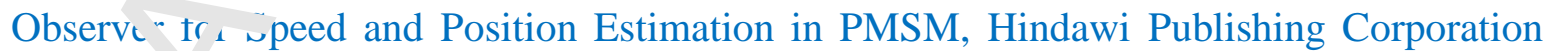
Mathematical Problems in Engineering Volume 2014,1-12. 
[41] D. Liang, J. Li, R. Qu, Super-Twisting Algorithm Based Sliding Mode Observer for Wide-Speed Range PMSM Sensorless Control Considering VSI Nonlinearity, IEEE International Electric Machines and Drives Conference (IEMDC), 2017

[42] J. J. Rath1, K. C. Veluvolu, M. Defoort,Y. C. Soh, Higher-order ,lidi ig mode observer for estimation of tyre friction in ground vehicles, IET Control Theory ^ ${ }^{\mathrm{p} p l .} 8$ (6) ( 2014), $399-408$.

[43] H. Rasmussen, P. Vadstrup, H. Borsting, Adaptive sen: orless ield oriented control of PM motors including zero speed, IEEE International syr pe sium Industrial Electronics, 2004 pp, $1191-1196$.

[44] H. Chaoui, P. Sicard, Adaptive Fuzzy Logic Cᄂ ^troı _. Permanent Magnet Synchronous Machines With Nonlinear Friction, IEEE Trans. Ind. Electron, 59 (February (2)) (2012), 1123-1133.

[45] N. Bounar, A. Boulkroune, F. Boudjema, M. M'Saad, M. Farza, Adaptive fuzzy vector control for a doubly-fed induction motor, 1 veurocomputing, 151 (March (Part 2)) (2015), 756-769.

[46] N. Öztürk , E. Çelik, Sp ed ontºl of permanent magnet synchronous motors using fuzzy controller based on ¿anetic algorithms, Electr. Power Energy Syst. 43 (2012) 889898.

[47] B. Belabbas, T. f llanui, ..1. Tadjine, M. Denai, High Order Sliding Mode Controller Simulation by a ${ }^{\mathbf{x}} /$ ina $^{\top}$, rbine for DFIG Protection against Overcurrent, electrotehcnica, electronica, aul matir a, 65(4) (2017) 142-147.

[48] S.M. Ki ‘raji, M I. B. B. Sharifian, Direct Thrust Force and Flux Control of a PM-Linear Synchr, nov, r.iotor Using Fuzzy Sliding-Mode Observer, Power Eng And Electr Eng, 13 (1) (2015) 1-9. 


\section{Conflict of Interest and Authorship Conformation Form}

Please check the following as appropriate:

- All authors have participated in (a) conception and de ion, or analysis and interpretation of the data; (b) drafting the article or "ev" sing it critically for important intellectual content; and (c) approval of the 1 . 9 l version.

- This manuscript has not been submitted to, no1 is un $r$ er review at, another journal or other publishing venue.

- The authors have no affiliation with any - " ar.adtion with a direct or indirect financial interest in the subject matter discusse 1 in the manuscript

- The following authors have affiliations ith organizations with direct or indirect financial interest in the su, ${ }^{\text {set }}$ matter discussed in the manuscript:

Author's name

Affiliation

Mansouri Mohamed Laboratory of En ${ }_{\mathrm{V}} \mathrm{E}$. gineering and Computer Engineering, IBN Khaldoun Uni ar ' $\mathrm{y}$, Tiaret, Algeria.

Hassaine Said Laboratory of $L^{n} \epsilon_{L_{\delta}}$, Ingineering and Computer Engineering, IBN Khaldoun Unı , rsity, Tiaret, Algeria.

Larbi Mhamed Laborator o1 _nergy Engineering and Computer Engineering, IBN Kha doun L aiversity, Tiaret, Algeria.

Bey Mohamed Labor ory or - nergy Engineering and Computer Engineering, IBN ha dou \& University, Tiaret, Algeria.

Allaoui Tayeb $\quad \mathrm{La}^{1}$ orato, ff Energy Engineering and Computer Engineering, I, $\mathrm{N}$ ' haldoun University, Tiaret, Algeria.

Denai Mouloud in hool of Engineering and Technology, University of $\mathrm{H}$ ' stfordshire, Hatfield, UK

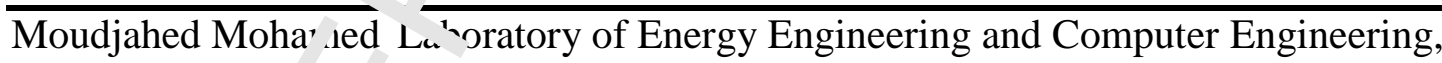
IB V Khaldoun University, Tiaret, Algeria. 